C-A/AP/\#404 Sept 2010

\title{
Booster and AGS Transverse Emittance During the 2006 and 2009 Polarized Proton Runs
}

K. Zeno

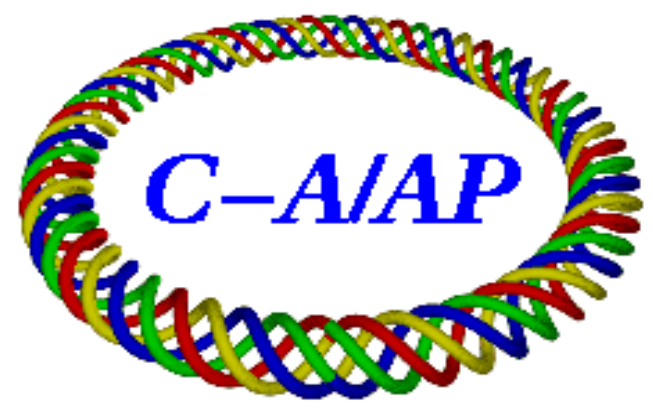

\section{Collider-Accelerator Department Brookhaven National Laboratory Upton, NY 11973}

Notice: This document has been authorized by employees of Brookhaven Science Associates, LLC under Contract No. DE-AC02-98CH10886 with the U.S. Department of Energy. The United States Government retains a non- 
Booster and AGS Transverse Emittance During the 2006 and 2009 Polarized Proton Runs

K. Zeno

April 23, 2010 


\section{Introduction}

This note is an overview of issues relating to transverse polarized proton emittance in the Booster and AGS. It also compares the transverse emittance during the FY'09 polarized proton run with it during the FY'06 run as several changes were made for the FY'09 run in an attempt to reduce the transverse emittance coming out of the AGS. The FY'06 run is used for comparison because it was relatively long, and it's believed that the performance of the injectors for polarized protons, up until FY'09, was best during that run.

Over the shutdown just before the FY'09 run work was done in LEBT and MEBT to reduce the emittance coming out of the Linac. Measurements of the beam coming out of Linac ${ }^{1}$ indicate that the horizontal normalized emittance was reduced from $11.0 \pi$ to $4.5 \pi \mathrm{mm} \mathrm{mrad}$, and that the vertical normalized emittance was reduced from $12.1 \pi$ to $5.5 \pi \mathrm{mm}$ mrad going from FY'06 to FY'09.

There were 2 new types of stripping foil installed in the Booster, called descriptively the 'strip' (\#6) and 'stamp' (\#2) foil, both nominally $100 \mu \mathrm{g} / \mathrm{cm}^{2}$. Both foils are composed of a diamond like material, and designed to reduce the number of times the beam goes through the foil. ${ }^{2}$ Other than those, there are two standard $100 \mu \mathrm{g} / \mathrm{cm}^{2}$ carbon foils (\#3 and 5), and one $200 \mu \mathrm{g} / \mathrm{cm}^{2}$ carbon foil (\#4). Of the two $100 \mu \mathrm{g} / \mathrm{cm}^{2}$ foils, one has shown some deterioration (\#3) in stripping efficiency.

During the FY'06 run a standard $100 \mu \mathrm{g} / \mathrm{cm}^{2}$ foil was generally used, and during the FY'09 run the strip foil was generally used, though the stamp foil was also used for the last 3-4 weeks of the run.

Both the FY'06 and FY'09 runs were about 5 months long, starting in late January, FY'06 ending in late June, and FY'09 ending in early July. A new injection setup was used for about the last 3 months of FY'09, from the beginning of April to the end of the run. This setup uses $1 / 2$ integer stopband correctors with the tunes near, but slightly greater than, 4.5 to distort the $\beta$ functions at the foil in order to reduce the emittance blowup caused by scattering on the foil.

New BtA quad settings, largely based on a MAD model of the BtA line and AGS were used for virtually the entire FY'09 run (Feb 5 to the end) in an attempt to improve the matching into the AGS. Since, it has been thought for some time that there is emittance blowup in the vertical due to an injection mismatch.

The nominal Linac pulse width was decreased from $400 \mu$ s to $300 \mu$ s in the latter part of february ' 09 , so the vast majority of the FY'09 run used a $300 \mu$ s pulse width. The

\footnotetext{
${ }^{1}$ D. Raparia, "Emittance and Foil" presentation March 23, 2009

${ }^{2}$ See "Minimizing Emittance Growth During H- Injection in the AGS Booster" K.A. Brown et al., PAC 09 for more details.
} 
standard pulse width for FY'06 was $400 \mu$ s. This has some implications for comparing the data from FY'06 with that from FY'09, but they don't seem insurmountable.

\section{Stripping Foils}

A comparison of stripping foils was made on April 21, 2009 using a $300 \mu$ s linac pulse. The BtA mutliwire MW006, 6 feet from the exit of the Booster, which has no quads between it and the Booster, was used to measure the emittance ${ }^{3}$ of the beam coming out of the Booster. The results are shown in Table I.

\begin{tabular}{|l|l|l|l|l|}
\hline Foil & $\# 2($ stamp $)$ & $\# 4(200 \mu \mathrm{g})$ & $\# 5(100 \mu \mathrm{g})$ & $\# 6($ strip $)$ \\
\hline H fwhm & $3.89 \mathrm{~mm}$ & $3.98 \mathrm{~mm}$ & $3.54 \mathrm{~mm}$ & $3.70 \mathrm{~mm}$ \\
\hline V fwhm & $6.03 \mathrm{~mm}$ & $7.21 \mathrm{~mm}$ & $5.75 \mathrm{~mm}$ & $6.38 \mathrm{~mm}$ \\
\hline H Emittance & $12.6 \pi$ & $13.1 \pi$ & $10.4 \pi$ & $11.4 \pi$ \\
\hline V Emittance & $5.6 \pi$ & $8.1 \pi$ & $5.1 \pi$ & $6.3 \pi$ \\
\hline H Amp/B input & 0.147 & 0.155 & 0.138 & $\mathbf{0 . 1 6 4}$ \\
\hline V Amp/B input & 0.093 & 0.084 & 0.083 & $\mathbf{0 . 0 9 5}$ \\
\hline B Early/B input & $91 \%$ & $101 \%$ & $81 \%$ & $102 \%$ \\
\hline
\end{tabular}

Table I: Comparison of the 4 types of stripping foils. Full width half max (fwhm) values are obtained from MW006 in BtA by using a gaussian fit on the beam profiles (rows 2 and 3). The Horizontal and Vertical Normalized 95\% emittances at MW006 are in rows 4 and 5 (see footnote 3 below). The ratios of the amplitudes of the fits to the Booster's injected intensity are in rows 6 and 7. The ratio of the intensity early in the Booster to that of the incoming beam is in row 7 (the absolute value of Booster input is not accurate on a few percent level but this isn't critical because changes to the ratio with different foils is what is relevant here).

As is evident from the table, the standard $100 \mu \mathrm{g} / \mathrm{cm}^{2}$ foil provides the smallest beam, and yields about $20 \%$ less beam than the $200 \mu \mathrm{g} / \mathrm{cm}^{2}$ foil. This is presumably because of the its poorer stripping efficiency. Curiously, the standard $100 \mu \mathrm{g} / \mathrm{cm}^{2}$ foil has, at least in previous runs, had a stripping efficiency only $15 \%$ less than that of the 200 $\mu \mathrm{g} / \mathrm{cm}^{2}$ foil. The standard $100 \mu \mathrm{g} / \mathrm{cm}^{2}$ foil was not used extensively this run, and often

${ }^{3}$ To find the emittance at the multiwire I use the gaussian fit in profile display to find the full width at half $\max$ ( $\mathrm{fwhm}$ ), then I use the formulas $\varepsilon \mathrm{h}_{95 \% \text { norm }}=0.83 * \mathrm{fwhm}^{2}$ and $\varepsilon \mathrm{V}_{95} \%$ norm $=0.155 * \mathrm{fwhm}^{2}$ to get the normalized $95 \%$ emittances. I derive these formulas in the following way, using $\beta_{\mathrm{x}}=3.0 \mathrm{~m}$ and $\beta_{\mathrm{y}}=16.0 \mathrm{~m}$ at the multiwire.. Since fwhm $=2.3548 \sigma$ and the $95 \%$ full width is $2 \sqrt{6} \sigma$. The ratio of $\Delta \mathrm{x} 95 \%$ (full width) to fwhm is $2 \sqrt{6} \sigma / 2.3548 \sigma=2.08$ and $\beta \gamma=2.3$. So I get, $\varepsilon h_{95 \% \text { norm }}=\left(\left(\frac{x_{95 \% \text { fullwidth }}}{2}\right)^{2} / \beta_{x}\right) \cdot \beta \gamma=\left(\left(\frac{2.08 f w h m}{2}\right)^{2} / 3.0 m\right) \cdot 2.3=0.83 \cdot f w h m^{2}$

for the horizontal, and by replacing $\beta_{\mathrm{x}}$ with $\beta_{\mathrm{y}}$ in the above formula I get $\varepsilon v_{95 \% \text { norm }}=0.155 \cdot f w h m^{2}$ for the vertical. 
significant tuning was required when switching from the strip or $200 \mu \mathrm{g} / \mathrm{cm}^{2}$ foil to get the injection efficiency to the nominal level. ${ }^{4}$ This behavior was new this year, and is not understood.

Though the strip foil is nominally $100 \mu \mathrm{g} / \mathrm{cm}^{2}$, its stripping efficiency is the same as the $200 \mu \mathrm{g} / \mathrm{cm}^{2}$, and the efficiency of the stamp foil is about halfway between the two (at least in this comparison). A $200 \mu \mathrm{g} / \mathrm{cm}^{2}$ foil is expected to have a stripping efficiency of $99.6 \%$, whereas a $100 \mu \mathrm{g} / \mathrm{cm}^{2}$ foil is expected to have a stripping efficiency of $91.3 \%{ }^{5}$ Yet the strip foil, which is nominally $100 \mu \mathrm{g} / \mathrm{cm}^{2}$ has the same stripping efficiency as the $200 \mu \mathrm{g} / \mathrm{cm}^{2}$ and the standard $100 \mu \mathrm{g} / \mathrm{cm}^{2}$ foil has normally had a stripping efficiency that is significantly worse than expected.

From a practical standpoint, the figure of merit for a foil is the brightness of the beam it provides. Though the stripping efficiency of the standard $100 \mu \mathrm{g} / \mathrm{cm}^{2}$ foil is significantly less than that of the $200 \mu \mathrm{g} / \mathrm{cm}^{2}$ foil it could still be preferable. Rows 6 and 7 in the table attempt to quantify this. They show the ratio of the gaussian fit's peak amplitude to the intensity of the injected beam (Booster Input), and that it is highest in both planes for the strip foil. In FY'06 the $100 \mu \mathrm{g} / \mathrm{cm}^{2}$ was slightly preferable to the 200 $\mu \mathrm{g} / \mathrm{cm}^{2}$ foil.

As the strip foil was the one used during most of the run, this comparison was done when injection had been optimized for it. Although effort was made to optimize for the other foils, most notably the stamp foil, this may take time, more time than was practical. The latter 3-4 weeks of the run was spent routinely using the stamp foil to see if its performance could be made as good or better than the strip foil. After running with it for weeks, a switch back to the strip foil was made and the injection efficiency was then the same as for the stamp foil, and the beam sizes on MW006 were nearly identical. Whether this is because the strip foil was no longer as optimized, the stamp foil was optimized better than it had been, or a combination of these effects is difficult to say because the day to day variations in injection efficiency, Booster input calibration, and BtA beam sizes are on the same order as the differences one would expect to see.

\section{The Effect of Linac Changes}

In order to see the effect of the Linac changes on the beam coming out of the Booster, one needs to compare the emittance during the FY'09 run with those in previous years under similar Booster conditions. Since we did not run with the $100 \mu \mathrm{g} / \mathrm{cm}^{2}$ foil for extended periods this year, and other things change from year to year, or even day to day, this comparison not completely fair. However, on Feb 3, 2009 profile measurements with the standard $100 \mu \mathrm{g} / \mathrm{cm}^{2}$ foil and a $400 \mu$ s pulse were made. These were the typical conditions for the FY'06 run. These measurements were made before the switch to halfinteger injection, so the injection setup was roughly similar to what it was in FY'06. Table II shows the results together with typical values taken from the FY'06 run.

\footnotetext{
${ }^{4}$ See Booster-AGS PP 2009 elog from Jan 15 between 1930 and 2130.

${ }^{5}$ D. Raparia, email communication, Jan 23, 2009
} 
The results show that in FY'06 the horizontal was significantly larger and the vertical was significantly smaller than when the FY'09 data was taken. To gauge whether there was any overall improvement I use the root mean square of the two emittances. ${ }^{6}$ For $\mathrm{FY}^{\prime} 06$ this is $14.4^{+} / 2.8 \pi \mathrm{mm} \mathrm{mr}$ and for FY'09 it is $10.8 \pi \mathrm{mm} \mathrm{mr}$. For an overall reduction in RMS emittance of $25 \%$ between FY'06 and FY'09. According to the emittance measurements made at Linac, the $\varepsilon_{R M S}$ for the beam coming from Linac went from $11.6 \pi \mathrm{mm} \mathrm{mr}$ to $5.0 \pi \mathrm{mm} \mathrm{mr}$, for a reduction of $57 \%$. Part of this discrepancy between the reduction in Linac emittance and Booster emittance is likely due to the emittance blowup due to scattering on the foil.

\begin{tabular}{|l|l|l|l|l|}
\hline & Horiz. fwhm & Vert. fwhm & Horiz. emittance & Vert. Emittance \\
\hline FY'06 & $4.77^{+} / .0 .58 \mathrm{~mm}$ & $6.70^{+} / \_.50 \mathrm{~mm}$ & $19.1^{+} / .4 .4 \pi \mathrm{mm} \mathrm{mr}$ & $7.0^{+} / .1 .02 \pi \mathrm{mm} \mathrm{mr}$ \\
\hline FY'09 & $3.77 \mathrm{~mm}$ & $7.85 \mathrm{~mm}$ & $11.8 \pi \mathrm{mm} \mathrm{mr}$ & $9.6 \pi \mathrm{mm} \mathrm{mr}$ \\
\hline
\end{tabular}

Table II: Comparison of FY '06 and FY'09 BtA MW006 beam sizes and emittances under similar conditions $\left(100 \mu \mathrm{g} / \mathrm{cm}^{2}\right.$ foil, $400 \mu$ s linac pulse, and standard injection setup). The data for FY'06 is an average of 4 profiles taken on 6 Feb, 13 Mar, 28 Mar, and 10 May 2006. The uncertainties for FY'06 come from these four data points.All data is without scraping in the Booster.

\section{Half Integer Injection}

In an attempt to reduce emittance growth due to scattering on the injection foil half integer injection was used. ${ }^{7}$ This appeared to improve the emittance coming out of the Booster significantly. Table III shows the values for emittances in both the normal injection and $1 / 2$ integer injection cases.

\begin{tabular}{|l|l|l|}
\hline & Fwhm & Emittance \\
\hline Normal & & \\
\hline Horizontal & $4.28^{+} / 0.11 \mathrm{~mm}$ & $15.0^{+} / .0 .78 \pi \mathrm{mm} \mathrm{mr}$ \\
\hline Vertical & $8.15^{+} / 0.39 \mathrm{~mm}$ & $10.3^{+} / .1 .00 \pi \mathrm{mm} \mathrm{mr}$ \\
\hline 1/2 Integer & & \\
\hline Horizontal & $3.59^{+} / 0.20 \mathrm{~mm}$ & $10.6^{+} / 1.19 \pi \mathrm{mm} \mathrm{mr}$ \\
\hline Vertical & $6.84^{+} / 0.45 \mathrm{~mm}$ & $7.3^{+} / .1 .00 \pi \mathrm{mm} \mathrm{mr}$ \\
\hline
\end{tabular}

TableIII: Comparison of BtA MW006 beam sizes and emittances during FY'09 with normal and $1 / 2$ integer injection. These measurements were taken with the strip foil and a pulse width of 300 $\mu$ s (the normal pulse width for most of the run). The data was taken over the course of the run. 20 profiles were used for the $1 / 2$ integer case, and 6 for the normal injection case. All data is without scraping in the Booster.

${ }^{6}$ That is, $\quad \varepsilon_{R M S}=\sqrt{\left(\varepsilon_{h}^{2}+\varepsilon_{v}^{2}\right) / 2}$

${ }^{7}$ See "Minimizing Emittance Growth During H- Injection in the AGS Booster" K.A. Brown et al., PAC 09 for more details. 
The results show a significant reduction in emittance in both planes. The RMS emittance was decreased by $29 \%$ in going from normal to $1 / 2$ integer injection.

Presumably this was due to less emittance blowup on the foil. This data was taken with a $300 \mu \mathrm{s}$ linac pulse, as that was the norm. As the horizontal and vertical tunes are both close to 4.5 and therefore close to each other, there is a large amount of coupling in and around injection. When a horizontal steering magnet in LtB is changed, the change in the emittance in BtA is usually more pronounced in the vertical plane, and vice versa. So, any conclusions about the emittances in either plane at injection from those in BtA are dubious.

\section{$300 \mu$ s vs. $400 \mu$ s Linac Pulse Width}

Towards the end of February the standard Linac pulse width was changed to 300 $\mu \mathrm{s}$ from $400 \mu \mathrm{s}$. The motivation for this was higher polarization from the OPPIS source because the laser pulse in the source is flatter over $300 \mu \mathrm{s}$ than over $400 \mu \mathrm{s} .^{8}$ But this further complicates the comparison between FY'06 and FY'09 because most of the data from FY'06 has a $400 \mu$ s pulse width and most of the data from FY'09 has a $300 \mu$ s pulse width.

Ideally, in order to compare FY'06 emittances with FY'09 after $1 / 2$ integer injection had been optimized, it is neceesary to compare data with the same linac pulse width and the same foil. Data exist for $1 / 2$ integer injection with a $300 \mu$ s pulse width and the standard $100 \mu \mathrm{g} / \mathrm{cm}^{2}$ foil, but not with a $400 \mu$ s pulse width. A pulse width scan from FY'06 shows that the widths on MW006 increase rather linearly with increasing pulse width (see figure 1).

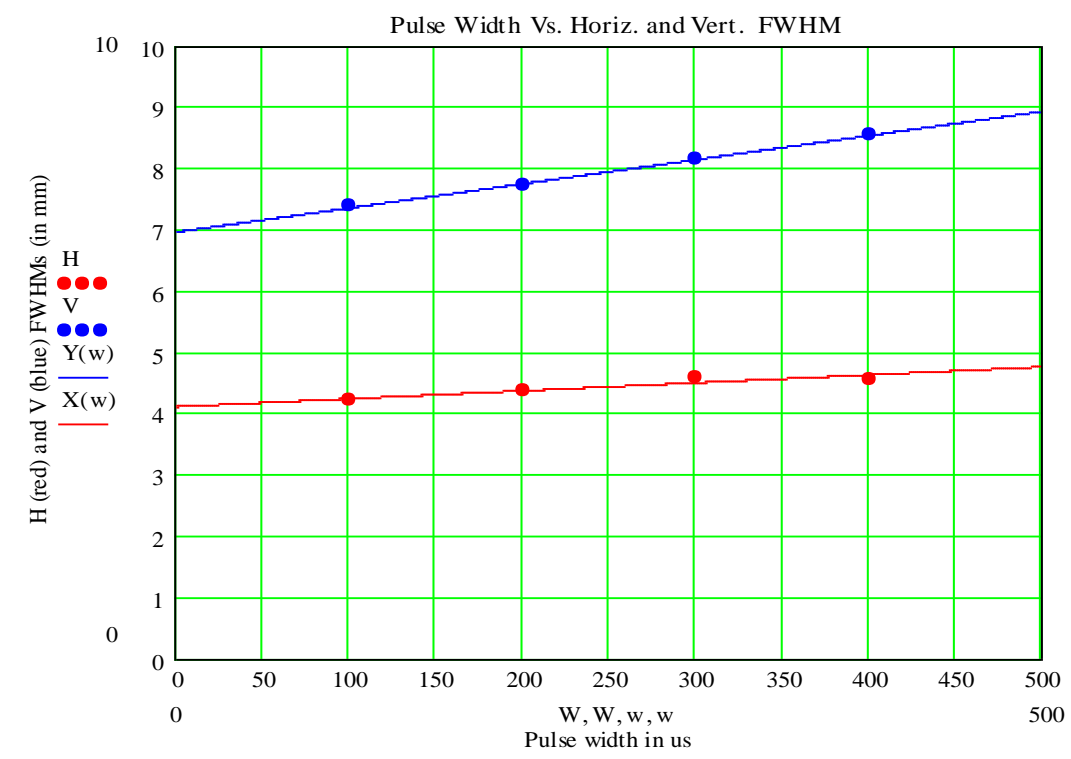

Figure 1: Linac Pulse width (in $\mu \mathrm{s}$ ) vs. Horizontal (red) and Vertical (blue) fwhms (in mm) on BtA multiwire MW006 from Feb. 82006 at 19:41, together with linear fits to data. Presumably with the $100 \mu \mathrm{g} / \mathrm{cm}^{2}$ foil.

${ }^{8}$ A. Zelenski 
There is data for $50 \mu$ s and $300 \mu$ s from FY'09 with $1 / 2$ integer injection and the $100 \mu \mathrm{g} / \mathrm{cm}^{2}$ standard foil. ${ }^{9}$ Table IV uses this data to estimate what the fwhms at MW006 would be for a $400 \mu$ s pulse from 2 sets of data, spaced a couple of weeks apart, for a 100 $\mu \mathrm{g} / \mathrm{cm}^{2}$ foil and $1 / 2$ integer injection. ${ }^{10}$

\begin{tabular}{|l|l|l|l|l|l|l|}
\hline & $\begin{array}{l}\text { FY'06, } \\
\text { 400 } \mathbf{~ m s}\end{array}$ & $\begin{array}{l}\text { FY'09a, } \\
\text { 300 ms* }\end{array}$ & $\begin{array}{l}\text { FY'09a, } \\
\text { 400ms est.* }\end{array}$ & $\begin{array}{l}\text { FY'09b, } \\
\text { 300ms** }\end{array}$ & $\begin{array}{l}\text { FY'09b, } \\
\text { 400 ms est.** }\end{array}$ & $\begin{array}{l}\text { Linac } \\
\text { beam }\end{array}$ \\
\hline H fwhm $(\mathrm{mm})$ & 4.77 & 3.36 & 3.43 & 3.54 & 3.61 & - \\
\hline V fwhm $(\mathrm{mm})$ & 6.70 & 6.17 & 6.46 & 5.75 & 6.04 & - \\
\hline $\begin{array}{l}\text { H emit. } \\
(\pi \text { mm mr })\end{array}$ & 19.1 & 9.4 & 9.8 & 10.4 & 10.8 & 5.5 \\
\hline $\begin{array}{l}\text { V emit. } \\
(\pi \text { mm mr })\end{array}$ & 7.0 & 5.9 & 6.5 & 5.1 & 5.7 & 4.5 \\
\hline $\begin{array}{l}\text { 'RMS' emit. } \\
(\pi \text { mm mr })\end{array}$ & 14.4 & 7.8 & 8.3 & 8.2 & 8.6 & 5.0 \\
\hline
\end{tabular}

Table IV: Comparison with $100 \mu \mathrm{g} / \mathrm{cm}^{2}$ foil of FY'06 data (same data as in table II) with 2 sets of FY'09 data with estimated widths for $400 \mu$ s Linac pulse (described above), and $1 / 2$ integer injection. $*$-April $8^{\text {th }}$ data. $* *$-April $21^{\text {st }}$ data.

Comparison of the $\varepsilon_{R M S}$ for the FY'09 data extended to $400 \mu$ s and the FY'06 data show that this value has been reduced by $40-42 \%$. The $\varepsilon_{R M S}$ coming from the Linac has been reduced by $57 \%$, but as the incoming beam is smaller in FY'09 than FY'06, the blowup on the foil should be a more significant component of the Booster emittance in FY'09, so one would not necessarily expect to see the same reduction in the Booster emittance as in the Linac emittance even if the beam was well matched in both cases.

As the $400 \mu$ s emittance was estimated from the $300 \mu$ s data, the $0 \mu$ s emittance, which should be the emittance without scattering from the foil, can be estimated from the $50 \mu \mathrm{s}$ fwhm data of April 8th. In this way the Linac emittance can be compared to a Booster emittance unaffected by the foil. The estimated $\varepsilon_{R M S}$ for a $0 \mu$ s pulse width is $6.65 \pi \mathrm{mm} \mathrm{mr}{ }^{11}$ The $\varepsilon_{R M S}$ of the Linac beam is $5.03 \pi \mathrm{mm} \mathrm{mr}$, which is $76 \%$ of the $0 \mu \mathrm{s}$ $\varepsilon_{R M S}$.

\footnotetext{
${ }^{9}$ Booster-AGS-pp elog, April 8, 2009, 1918 and 1919 entries. In going from a $50 \mathrm{~ms}$ pulse to a $300 \mathrm{~ms}$ pulse the $(\mathrm{h}, \mathrm{v})$ fwhms from $(3.18 \mathrm{~mm}, 5.45 \mathrm{~mm})$ to $(3.36 \mathrm{~mm}, 6.17 \mathrm{~mm})$ for the $100 \mu \mathrm{g} / \mathrm{cm}^{2}$ foil. Assuming the increase is linear, the rate of change of the fwhm on MW006 is $0.072 \mathrm{~mm} / 100 \mathrm{~ms}$ for the horizontal, and $0.288 \mathrm{~mm} / 100 \mathrm{~ms}$ for the vertical.

${ }^{10}$ Booster-AGS-pp elog, April 21, 2009, 2153 entry.

$1150 \mu \mathrm{s}(\mathrm{h}, \mathrm{v})$ fwhms $=(3.18,5.45) \mathrm{mm}->0 \mu \mathrm{s}$ fwhms $=(3.14,5.31)->(\mathrm{h}, \mathrm{v}) 95 \%$ normalized emittances of $(8.2,4.6) \pi \mathrm{mm} \mathrm{mr}->\varepsilon_{R M S}=6.65 \pi \mathrm{mm} \mathrm{mr}$.
} 


\section{The Effect of Dispersion on the Measured Emittance}

Since the horizontal (estimated) emittance measured in BtA is significantly larger than what's measured from Linac, but the vertical agrees fairly well, I thought it worthwhile to consider the effect the momentum spread of the beam at MW006 has on the fwhms measured there, and thereby the value for the emittance that is obtained.

Using the AgsModelViewer program I obtained a rough estimate for the $\Delta \mathrm{p} / \mathrm{p}$ of the beam at extraction of 2.85e-3. ${ }^{12}$ The dispersion value at MW006 that I use is $0.585 \mathrm{~m} .{ }^{13}$ This gives a dispersion component of the full beam width at MW006 of 1.67 $\mathrm{mm}$. For the $0 \mu \mathrm{s}$ fwhm of $3.14 \mathrm{~mm}$ this means that the optical component of that fwhm is $2.94 \mathrm{~mm} .{ }^{14}$ This gives a $0 \mu \mathrm{s} 95 \%$ normalized horizontal emittance of $7.2 \pi \mathrm{mm} \mathrm{mr}$, and reduces the estimated $0 \mu \mathrm{s} \varepsilon_{R M S}$ from 6.65 to $6.03 \pi \mathrm{mm} \mathrm{mr}$. The measured Linac RMS emittance is $5.03 / 6.03=83 \%$ of this value, up from $76 \%$ when the momentum spread is not considered.

\section{Normal Running Conditions in FY'06 and FY'09}

Table V shows the normal BtA MW006 beam sizes, without scraping, during FY'06 and FY'09. Although the pulse width for FY'09 was normally $100 \mu$ s shorter than in FY'06, this was offset somewhat as the stripping efficiency of the strip foil is about $15 \%$ higher than the standard $100 \mu \mathrm{g} / \mathrm{cm}^{2}$ foil used in FY'06.

\begin{tabular}{|c|c|c|c|}
\hline$F^{\prime} 06(100 \mu g, 400 \mu s)$ & fwhm & 95\% Norm Emit. & RMS Emittance \\
\hline Horizontal & $4.77^{+} / .0 .58 \mathrm{~mm}$ & $19.1^{+} / .4 .4 \pi \mathrm{mm} \mathrm{mr}$ & \multirow[t]{2}{*}{$14.4^{+} / 2.8 \pi \mathrm{mm} \mathrm{mr}$} \\
\hline Vertical & $6.70^{+} / .0 .50 \mathrm{~mm}$ & $7.0^{+} / 1.0 \pi \mathrm{mm} \mathrm{mr}$ & \\
\hline \multicolumn{4}{|l|}{$\begin{array}{l}\text { FY'09 (strip foil, } 1 / 2 \\
\text { integer inj., } 300 \mu \mathrm{s}, \\
\text { New Linac optics) }\end{array}$} \\
\hline Horizontal & $3.59^{+} / .0 .20 \mathrm{~mm}$ & $10.7^{+} / 1.19 \pi \mathrm{mm} \mathrm{mr}$ & \multirow[t]{2}{*}{$9.2^{+} / .0 .9 \pi \mathrm{mm} \mathrm{mr}$} \\
\hline Vertical & $6.84^{+} / .0 .45 \mathrm{~mm}$ & $7.3^{+} / 1.00 \pi \mathrm{mm} \mathrm{mr}$ & \\
\hline
\end{tabular}

Table V: Beam sizes and emittances at BtA MW006 for the standard injection setups in FY'06 and FY'09 (no scraping). FY'06 data is the same as in Table II.

\footnotetext{
${ }^{12}$ I use values of $150 \mathrm{~ns}$ for the full width of the bunch (typical width measured for the first turn in the AGS using the wall current monitor), $23 \mathrm{kV} /$ turn for the extraction voltage (the programmed value), and an $\mathrm{dB} / \mathrm{dt}$ of $50 \mathrm{~g} / \mathrm{ms}$.

${ }^{13}$ Vincent Schoeffer, from MAD model.

${ }^{14}$ The ratio of the $95 \%$ full width to the fwhm is 2.08 , and $3.14 \mathrm{~mm}$ is the measured fwhm, so $\sqrt{(2.08 * 3.14)^{2}-1.67^{2}}=6.314 \mathrm{~mm}$ is the opitcal component of the $95 \%$ full width. The ratio of $95 \%$ full width to fwhm is $2 \sqrt{6} / 2.35=2.085$, so the optical fwhm is $6.134 / 2.085=2.94 \mathrm{~mm}$.
} 


\section{AGS Emittance}

The IPM emittances on the AGS flattop with the Rf disabled are the numbers quoted for the AGS emittances. In FY'06 typical emittances were 12 (horiz.) and 14-15 (vert.) $\pi \mathrm{mm}$ mrad for $14-15 \mathrm{e} 10$ late in the AGS ${ }^{15}$. For FY'09 these emittances were perhaps slightly smaller, more like 10-11 (horiz.) and 14-15 $\pi \mathrm{mm} \mathrm{mrad}$ (vert.) for 14$15 \mathrm{e} 10$ late in the $\mathrm{AGS}^{16}$. Given that the BtA emittance, at least for unscraped beam and in the horizontal is smaller in FY'09, why is the horizontal only slightly smaller late in the AGS? Also, why in both cases is the vertical in AGS so much larger than in BtA?

\section{Comparing the Two Runs with similar AGS Late Intensities}

The BtA and IPM emittances for more or less standard running conditions in FY'06 and FY'09 and similar AGS late intensities are shown in Table VI. Since these are standard running conditions the data is with Booster vertical scraping, which is why there is such a discrepancy between the Booster early and late intensities.

\begin{tabular}{|l|l|l|l|l|l|l|l|}
\hline & B input & B early & B late & 1 xfer & Cbm & late & B late/B early \\
\hline Fy06 & 45 & 34 & 18 & 17 & 15.5 & 14.3 & 0.53 \\
\hline Fy09 & 33.1 & 31.8 & 17.8 & 17.8 & 16.6 & 14.1 & 0.56 \\
\hline
\end{tabular}

\begin{tabular}{|l|l|l|l|l|l|l|}
\hline & $\begin{array}{l}\text { BtA H } \\
\text { emit }\end{array}$ & $\begin{array}{l}\text { IPM H } \\
\text { emit }\end{array}$ & $\begin{array}{l}\text { BtA V } \\
\text { emit }\end{array}$ & $\begin{array}{l}\text { IPM V } \\
\text { emit }\end{array}$ & $\begin{array}{l}\text { BtA RMS } \\
\text { emit }\end{array}$ & $\begin{array}{l}\text { IPM RMS } \\
\text { emit }\end{array}$ \\
\hline Fy06 & 14.8 & 10.8 & 3.3 & 14.8 & 10.7 & 13.0 \\
\hline Fy09 & 9.7 & 9.8 & 2.9 & 13.0 & 7.2 & 11.5 \\
\hline Fy06/Fy09 & 0.66 & 0.91 & 0.88 & 0.88 & 0.67 & 0.89 \\
\hline
\end{tabular}

Table VI: Data for similar AGS late intensities in FY'06 and FY'09. May 1, 1208:24 for IPM and intensities as well as 1211:51 for MW006 profiles $(\mathrm{h}, \mathrm{v})=(4.22,4.61) \mathrm{mm}$. May 19, 2009 1701:56 for IPM and intensities, and 1701:03 for MW006 profiles $(\mathrm{h}, \mathrm{v})=(3.41,4.30) \mathrm{mm}$.

It is important to note that running conditions change considerably from day to day, and on a level relevant to this comparison. Nevertheless, there are some notable differences, most glaring is the amount of beam coming from Linac that produces more or less the same early intensity in the Booster. If the FY'09 Booster input is multiplied by $4 / 3$ (since we ran with a $400 \mu$ s pulse in FY'06 and a $300 \mu$ s pulse in FY'09) it is quite close to the FY'06 Booster input, so the linac current, at least in these 2 cases appears to be similar. If one accounts for the $15 \%$ difference in stripping efficiency though, the injection efficiency for the FY'06 data is $34 /\left(45^{*} 0.85\right)=89 \%$. In the FY' 09 case it is $31.8 / 33.1=96 \%$. A variation of this magnitude in injection efficiency from day to day in either run was not unusual, and may be due, at least in part, to fluctuations in the HEBT transformer calibration.

\footnotetext{
${ }_{15}^{15}$ See for example, May 11, 2006 Booster-AGS pp elog entry at 1558 (the value at $810 \mathrm{~ms}$ is used).

${ }^{16}$ See for example, May 14, 2009 Booster-AGS pp elog entry at 1754
} 
Given that the strip foil creates a somewhat larger beam than the $100 \mu \mathrm{g} / \mathrm{cm}^{2}$ foil, the fact that the emittances in BtA are smaller in FY'09 than FY'06 is significant considering that there was perhaps slightly less scraping in the FY'09 case than in the FY'06 case (as evidenced by the ratio Booster late/Booster early). So, with $3 / 4^{\text {th }}$ of the FY'09 Linac intensity the same amount of beam is available at Booster extraction, and it is smaller, particularly in the horizontal. The ratio of the RMS emittances in BtA of the 2 cases (FY'06/FY'09) is 0.67 . Yet, the ratio of the RMS emittances as measured by the IPM is 0.89. So, although there seems to be a significant reduction in emittance in BtA, the change is not reflected much in the IPM measurement on the AGS flattop.

The FY'06 data for the unscraped (table V) and normal running scraped (table VI) cases show that the horizontal emittance goes from 19.1 to $14.8 \pi \mathrm{mm} \mathrm{mr}$, and the vertical from 7.0 to $3.3 \pi \mathrm{mm} \mathrm{mr}$. In FY'09 the horizontal emittance goes from 10.7 to $9.8 \pi \mathrm{mm} \mathrm{mr}$, and the vertical from 7.3 to $2.9 \pi \mathrm{mm} \mathrm{mr}$ for these 2 cases. The FY'06 RMS emittance was reduced $38 \%$, in FY'09 it was reduced $33 \%$ between unscraped and scraped cases. The amount of scraping, judging from the early and late intensities in table VI was slightly greater in the FY'06 case.

\section{Comparing BtA and IPM Emittances}

The ratio of the horizontal AGS flattop and BtA emittances was 0.74 in FY'06 and 1.01 in FY'09. This ratio for the vertical is 4.5 for both sets of data. Although the horizontal ratio shows some interesting behavior (i.e.- the emittance was somewhat smaller at AGS flattop than in BtA in FY'06), the most glaring discrepancy is in the vertical.

In order to try to understand what may be the cause for this large discrepancy in the vertical, it may be useful to look at some data that was taken for the IPM and BtA MW006 where the AGS intensity was varied by adjusting the amount of vertical scraping.

The IPM measurement gives a higher value for the emittance on the AGS flattop when there is Rf voltage present and the beam is bunched. Typically, the quoted values are for the case where the Rf has been turned off on the flattop. This dependence of the emittance value on the $\mathrm{Rf}$ voltage seems to scale with voltage amplitude, and be related to the longitudinal bunch width, so that there is less of an effect when the Rf voltage is lower. The Rf voltage is about $20 \%$ of its flattop value near injection and the bunches are much wider as well (150 ns vs. $30 \mathrm{~ns}$ ), hence this effect is much less there than on flattop.

Figure 2 shows the vertical BtA emittance as well as the vertical IPM emittance shortly after injection as a function of AGS late intensity. There was Rf voltage present for this set of measurements, but for the reason described above its effect on the measured emittance is neglected here (the presence of this Rf voltage would tend to increase the values for emittance that the IPM program reports). Additionally, the IPM program uses a value for the $\beta$ function at the IPM to calculate the emittance. This value is for the bare AGS, it does not take into account the presence of the Siberian Snakes. 
Although the model of the AGS with the snakes on is not well established, if it is used the value for the $\beta$ function at the vertical IPM changes from $22 \mathrm{~m}$ to $15 \mathrm{~m} .{ }^{17}$ This is taken into account in figure 2 .

From the figure it appears that in both measurements the emittance grows rather linearly with AGS late intensity, so I fit the data to straight lines. The slope of the lines is the same, the difference is only in their y-intercepts.

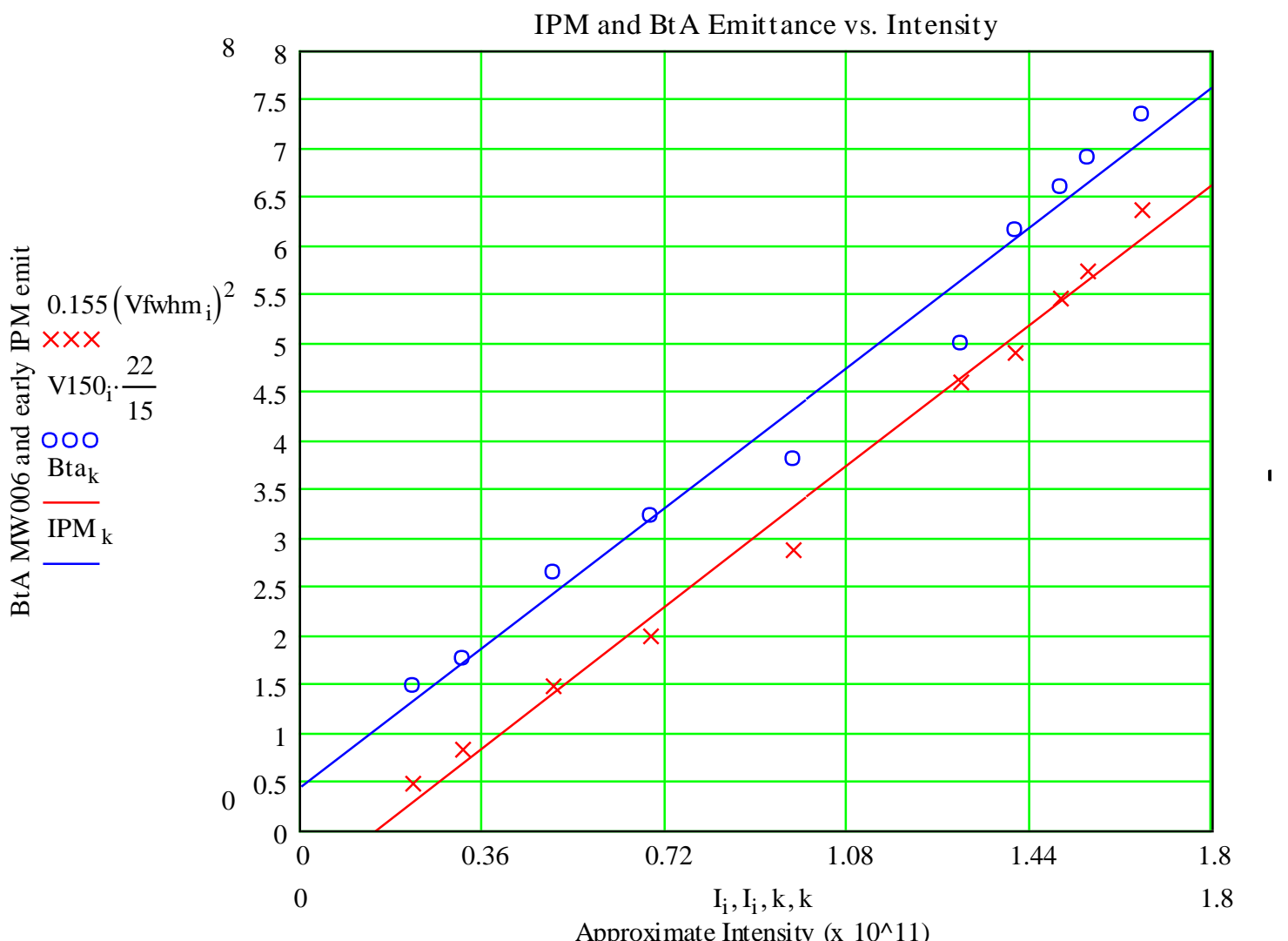

Figure 2: BtA MW006 (red x's) and AGS IPM (blue o's) vertical 95\% normalized emittance data about $10 \mathrm{~ms}$ after injection vs. AGS late intensity adjusted by varying the vertical scraping in Booster. Also shown are linear fits of the data. For the BtA data the fit is Emit=4.01*Intensity-0.59 and for the IPM data it is Emit=4.00*Intensity+0.44. Data taken from April 4, 2009 Booster-AGS PP elog.

Figure 3 shows data for the same IPM and emittance measurements, but this time the IPM data is taken on the AGS flattop with the Rf disabled. At high energy the effect of the snakes on the $\beta$ functions at the IPMs is greatly reduced so the $\beta$ functions used by the IPM program should be nearly correct. The IPM data at flattop seems pretty linear too, except for the 3 points at the highest intensities where it seems to increase at a higher rate, possibly indicating some intensity dependent emittance blowup. So, once again I fit

\footnotetext{
${ }^{17}$ K.A. Brown, Booster-AGS PP 2009 elog, April $21^{\text {st }}$ 19:56 entry by shows the $\beta$ functions at the IPMs obtained from the online model as a function of time in the cycle. The IPM measurement taken near injection was at about AT0+150ms.
} 
the IPM data to a straight line, but this time excluding the last three points in the fit. In this case, the slope of the IPM line is greater than the BtA line, 5.64 vs. 4.01, but most of the discrepancy is still in the y-intercept. The y-intercept for the IPM data has gone from 0.44 near injection to 5.61 on the flattop. If the emittance as a function of intensity varied by scraping were roughly linear, one would expect that the y-intercept would be close to zero as it is for the BtA measurement.

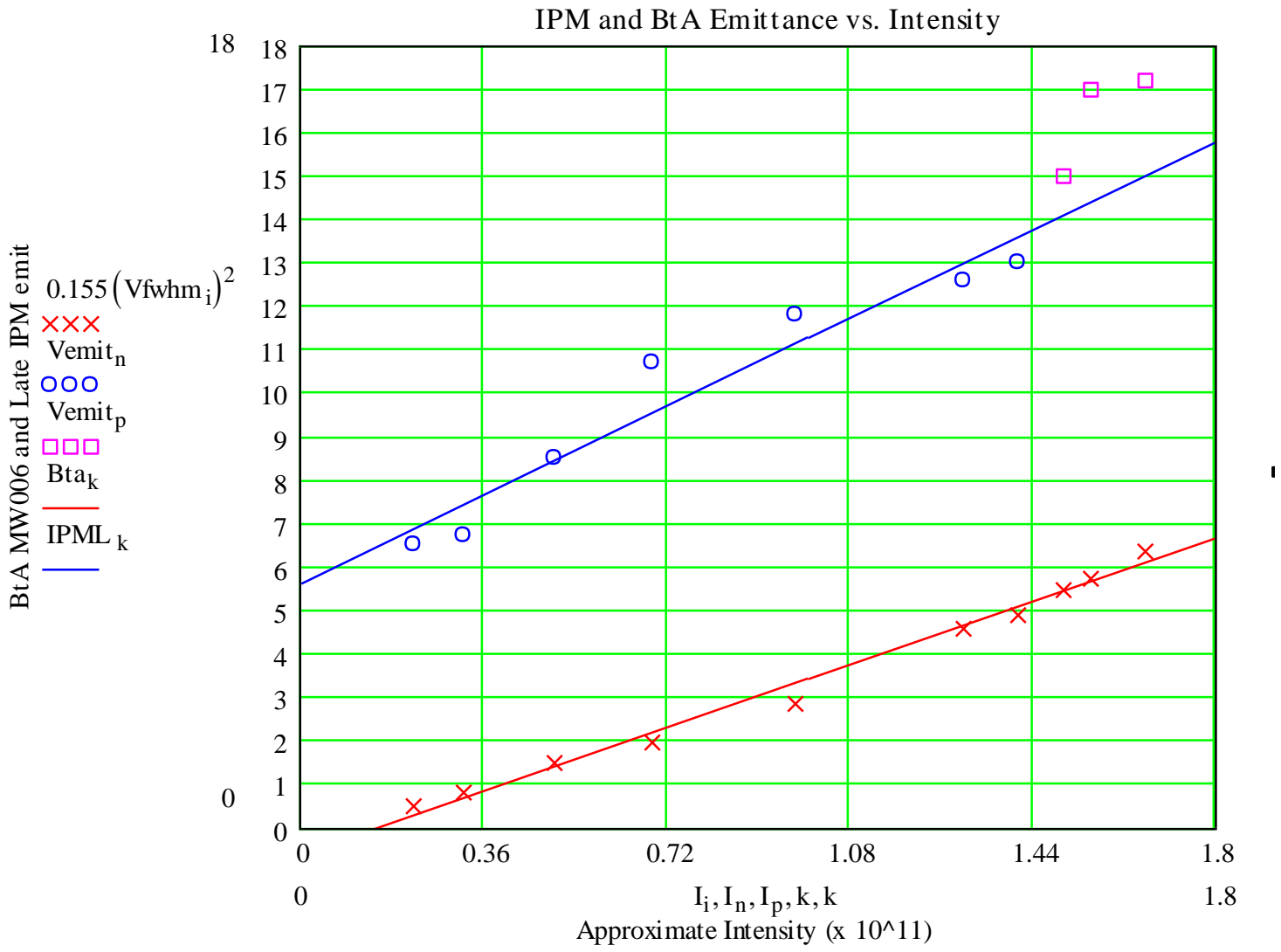

Figure 3: BtA MW006 (red x's) and AGS IPM (blue o's and magenta squares) vertical 95\% normalized emittance data on the AGS flattop vs. AGS late intensity adjusted by varying the vertical scraping in Booster. Also shown are linear fits of the data. For the BtA data the fit is Emit=4.01*Intensity-0.59 and for the IPM data it is Emit=5.64*Intensity+5.61. The linear fit for the IPM data does not include the 3 data points at the highest intensites. Data taken from April 4, 2009 Booster-AGS PP elog.

\section{BtA Matching and the A15 Multiwire}

The A15 multiwire in the AGS ring is used to measure beam profiles in the AGS at injection. As with BtA MW006, the profiles are fit to gaussians. By killing the beam on the first turn around the AGS single turn profiles of the beam at A15 are obtained. Multiple turn profiles can also be obtained by allowing the beam to circulate for many turns until it is gradually lost from scattering on the multiwire. 
An increase in the vertical beam size is typically observed at the A15 multiwire between the first turn and multiple turn cases. This effect is generally not as pronounced in the horizontal plane. This increase in the vertical width measured at the multiwire is normally thought to be due to an injection mismatch. Since the vertical emittance, at least on the flattop, reported by the IPM is much larger than that reported by BtA MW006, it is thought that this mismatch may be at least partly responsible.

If the emittance increase observed on the IPM were solely associated with a mismatch then the ratio of both of the IPM measurements to the BtA measurement should be equal to the emittance blowup at injection. Figure 4 shows the ratios of the flattop and near injection IPM to BtA measurements. In both cases the ratios decrease with increasing intensity and larger incoming emittance. The deviation from a flat line is much greater in the flattop case. But if an injection mismatch were largely responsible, then why is the IPM data near injection in such better agreement with the BtA data?

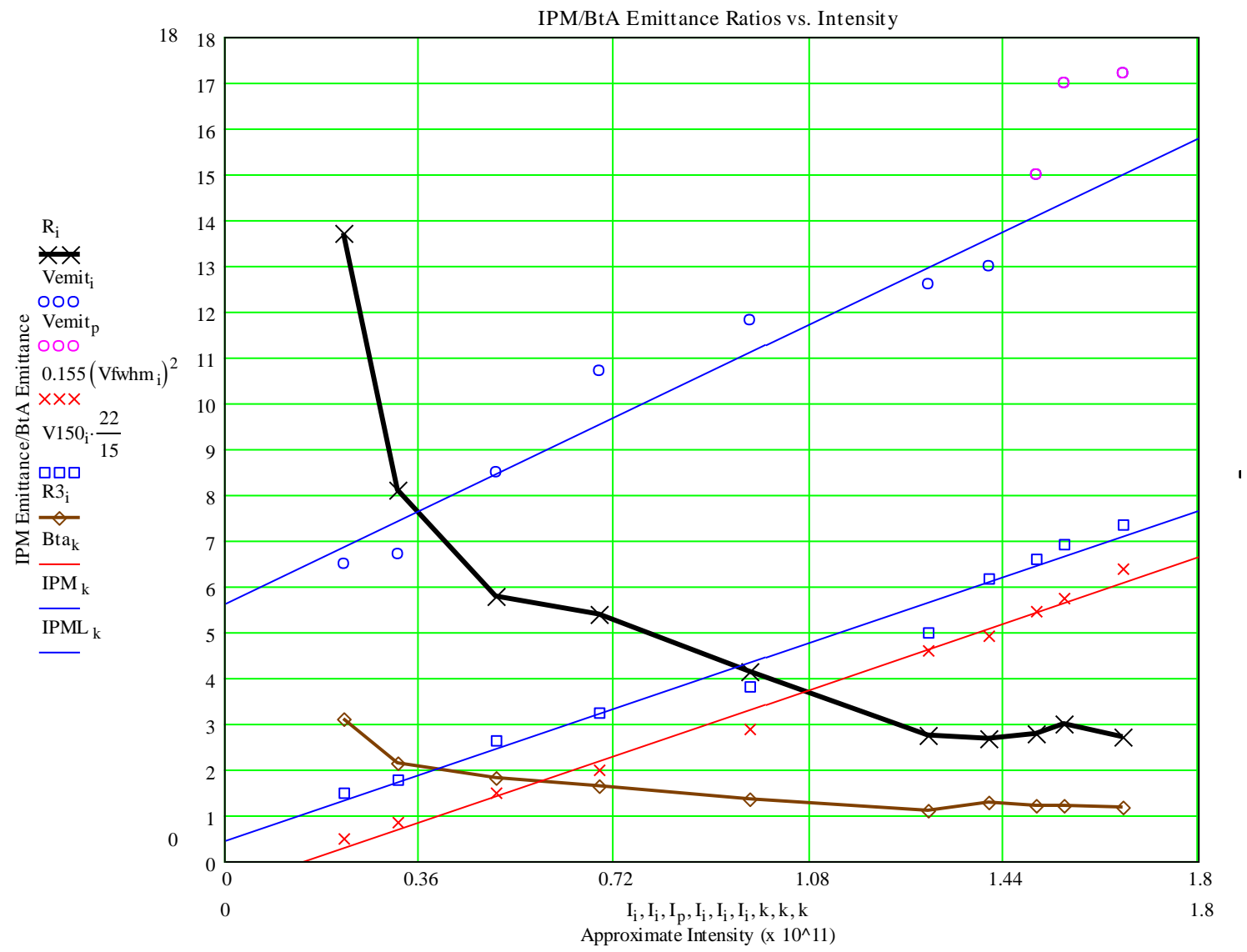

Figure 4: Ratio of IPM vertical Emittance to BtA MW006 vertical emittance. The black curve is the ratio of the flattop IPM to BtA emittance data. The brown curve is the ratio of the early IPM to BtA emittance data. The other data and lines are as in figures 2 and 3.

There are other mechanisms besides injection mismatch that can cause emittance blowup. Sometimes the blowup is intensity dependent, and it seems that this kind of effect is occuring for the flattop emittance at intensities higher than about $1.5 \mathrm{e} 11$ (magneta o's). If this were the effect that was causing the increased emittance in AGS at 
the other intensities then one would expect it to be worse at higher intensities, but the opposite is true. Stopbands and other resonant phenomena can also cause blowup at low intensity, but that blowup does not usually become smaller as the intensity is increased.

An argument raised in support of an injection mismatch causing the emittance blowup observed on the IPM, is that at flattop with small beam sizes the bin spacing is not small enough, and so there is some minimum emittance that the IPM will report. As the beam size increases the measurement becomes more accurate. So that the measurements at higher intensities (and larger beam sizes) are more representative.

Modeling the way the IPM data is binned and fit to a Gaussian shows that there is more or less a constant offset between the actual emittance at a particular energy and the emittance measured by the IPM. But the offset, in the case of flattop energy, is on the order of $1 \pi \mathrm{mm} \mathrm{mr}$, not $6 \pi \mathrm{mm} \mathrm{mr}$. ${ }^{18}$ The offset is about $0.1 \pi \mathrm{mm} \mathrm{mr}$ at injection energy. So although the offset is expected to be significantly larger at extraction energy than injection energy, which is similar to what is observed, the magnitude is wrong.

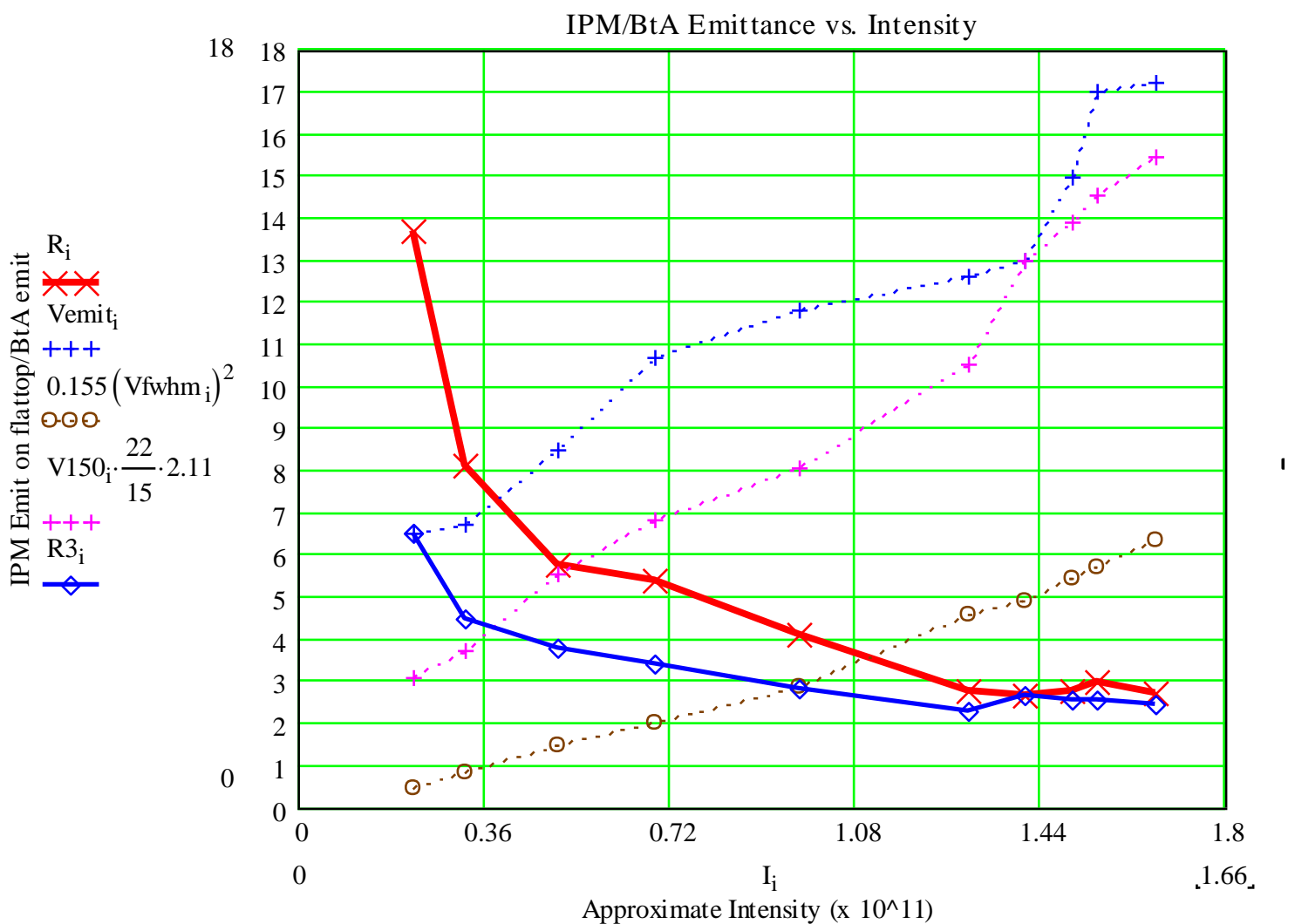

Figure 5: Same as figure 4 except using a $\beta$ function $(\beta=46.4 \mathrm{~m})$ for the near injection IPM data that causes the near injection and flattop data to agree at an AGS late intensity of about $1.4 \mathrm{e} 11$. The red curve is the ratio of the flattop IPM to BtA emittance data. The blue curve is the ratio of

\footnotetext{
${ }^{18}$ L. Ahrens, "Measuring the Transverse Beam Size of the Beam with the AGS IPM: A Simple Exercise", 27 Aug 09.
} 
the early IPM to BtA emittance data. Blue +'s are flattop data, magenta +'s are near injection IPM data (using b=46.4 m), and brown o's are BtA MW006 data.

If one assumes that the emittances given by the IPM are accurate, and there is little vertical emittance blowup during acceleration, than how can the emittance near injection be so different than on flattop? One can argue that the near injection measurement is wrong because the $\beta$ function used is incorrect. So, if one allows the $\beta$ function to vary its value would need to be about $46 \mathrm{~m}$ (instead of the model prediction of $22 \mathrm{~m}$ ) in order for the emittances at an intensity of 1.4e11 to agree (I use that intensity because it is just below some nonlinearity in the emittance data that may be indicative of blowup during acceleration), and a significant 'offset' would still exist for lower intensities (see figure 5) which is hard to reconcile with the flattop data.

Alternately, if the $\beta$ function were increased further so that the near injection and flattop emittances agreed at the lowest intensities $(\beta=98 \mathrm{~m})$, then the slope of the near injection data would be about 3 times greater than that of the flattop data, something which doesn't lend itself to a physical interpretation.

\section{Using the Sum of Two Gaussians to Fit A15 Profiles}

Multi-turn A15 multiwire profiles show evidence of the superpositioning of two gaussian shaped beams that does not exist in the single turn case. Figure 6 illustrates this by showing the first and multi-turn cases using a beam that has been scraped both horizontally and vertically in the Booster, so that the first turn profiles may have smaller tails than that of a gaussian. The Gaussian fits that the ProfileDisplay performs are shown together with the data.

Regardless of the mechanism responsible for the shape of the profiles, fitting the multi-turn data to the sum of two gaussians gives a fit which agrees very well with the data (yet using the sum of three gaussians adds nothing). Figure 7 shows the multi-turn data for each plane, together with fits to the sum of two gaussians, as well as the two gaussians themselves. The $\sigma$ values of the narrow, $\sigma_{\mathrm{n}}$, and wide, $\sigma_{\mathrm{w}}$, gaussians and the $\sigma$ of the single, $\sigma_{s}$, gaussian (from ProfileDisplay) fits are shown in table VII. The ratios

$\sigma_{\mathrm{n}} / \sigma_{\text {singleturn }}$ and $\sigma_{\mathrm{s}} / \sigma_{\text {singleturn, }}$, where $\sigma_{\text {singleturn }}$ is the single turn $\sigma$ (from ProfileDisplay) are also shown. The values of $\sigma_{\mathrm{n}} / \sigma_{\text {singleturn }}$ are closer to one than the values for $\sigma_{\mathrm{s}} / \sigma_{\text {singleturn }}$, which is the value one would expect if injection were matched.

Figure 8 shows the case where the beam coming from the Booster has not been scraped. In this case, the tails on the multi-turn profiles are not as pronounced as in the case with the scraped beam, but the double gaussian fits are still better than the single gaussian ones. Table VIII shows the $\sigma$ values, $\sigma_{\mathrm{n}} / \sigma_{\text {singleturn }}$, and $\sigma_{\mathrm{s}} / \sigma_{\text {singleturn }}$ for the fits associated with this data. 
Single turn, heavily scraped


Multi-turn, of same beam
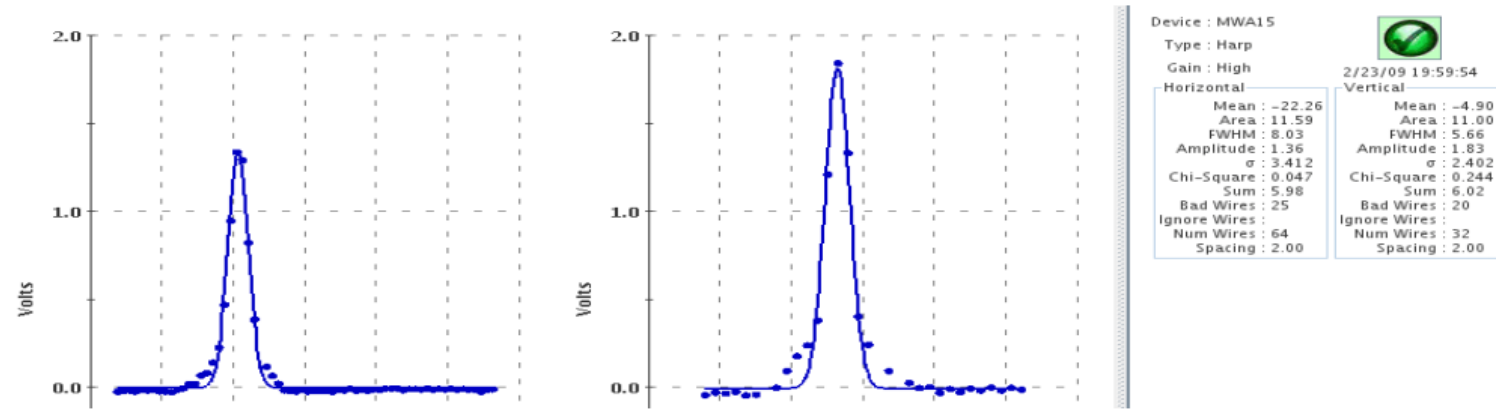

Figure 6: Single turn and Multi-turn A15 Multiwire data (dots) and gaussian fits (curves) for beam scraped in both planes in the Booster. Data from 23 Feb 09 19:57 and 19:59 respectively.
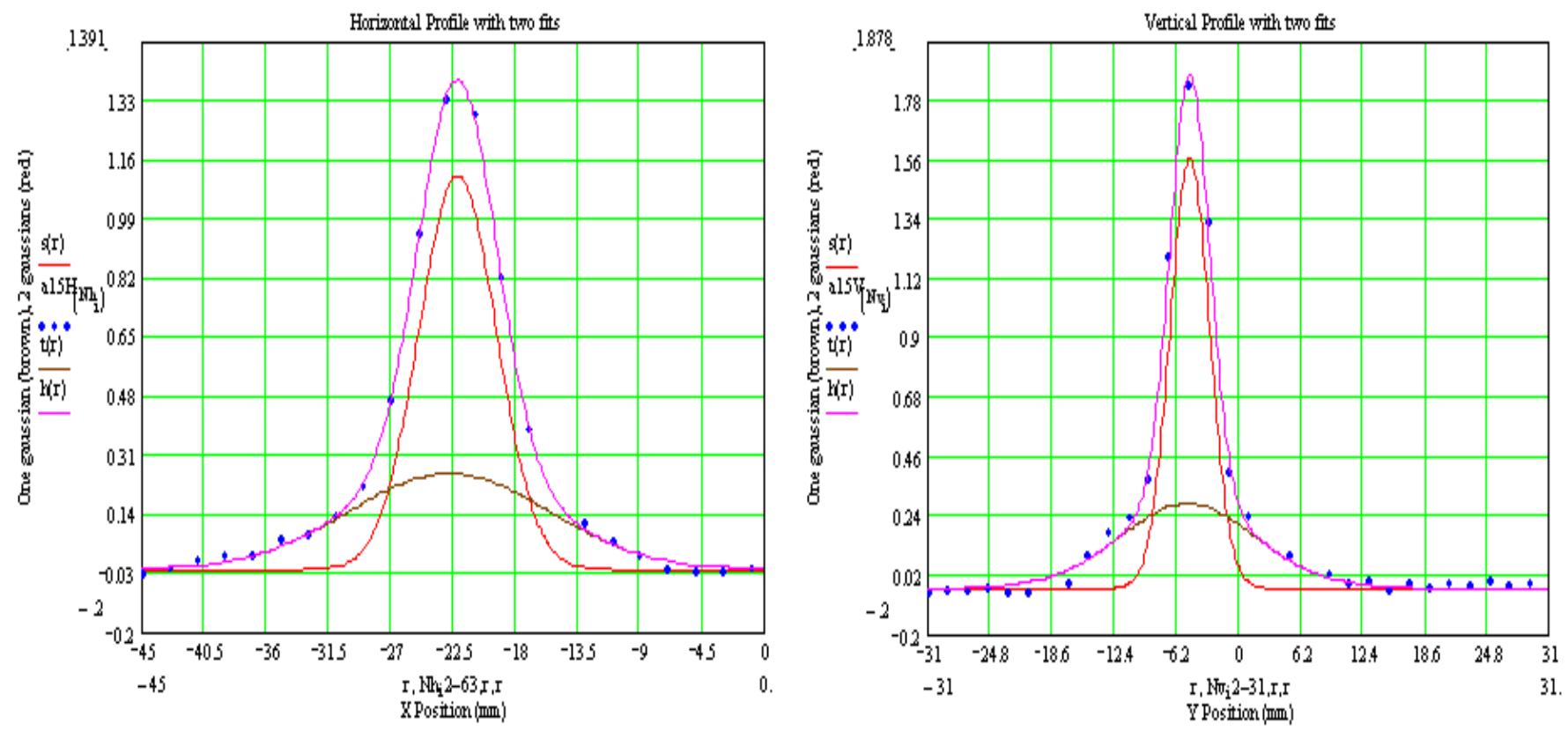

Figure 7: Horizontal (left) and vertical (right) A15 Multiwire data (blue dots) and fits to the sum of two gaussians (magenta curves) for the multi-turn profiles in figure 6 . The two gaussians themselves are also shown (red and brown curves). 


\begin{tabular}{|l|l|l|}
\hline Case & Horizontal & Vertical \\
\hline Single turn $\sigma\left(\sigma_{\text {singleturn }}\right)$ & $2.75 \mathrm{~mm}$ & $1.62 \mathrm{~mm}$ \\
\hline Single Gaussian, multi-turn $\sigma\left(\sigma_{\mathrm{s}}\right)$ & $3.41 \mathrm{~mm}$ & $2.38 \mathrm{~mm}$ \\
\hline Double Gaussian fit, narrow Gaussian for multi-turn $\sigma\left(\sigma_{\mathrm{n}}\right)$ & $2.80 \mathrm{~mm}$ & $1.96 \mathrm{~mm}$ \\
\hline$\left(\sigma_{\mathrm{s}} / \sigma_{\text {singleturn }}\right)$ for single gaussian fit & 1.23 & 1.48 \\
\hline$\left(\sigma_{\mathrm{n}} / \sigma_{\text {singleturn }}\right)$ for double gaussian fit & 1.0 & 1.23 \\
\hline Narrow Gaussian Area/Wide Gaussian Area & 1.59 & 1.39 \\
\hline
\end{tabular}

Table VII: Gaussian fit $\sigma$ values $\sigma_{\text {singleturn }}, \sigma_{\mathrm{s}}, \sigma_{\mathrm{n}}$, as well as $\sigma_{\mathrm{n}} / \sigma_{\text {singleturn, }}$, and $\sigma_{\mathrm{s}} / \sigma_{\text {singleturn }}$, and the ratio of narrow to wide gaussian areas for the A15 multiwire profiles in figure 6 .

Single Turn, No scraping
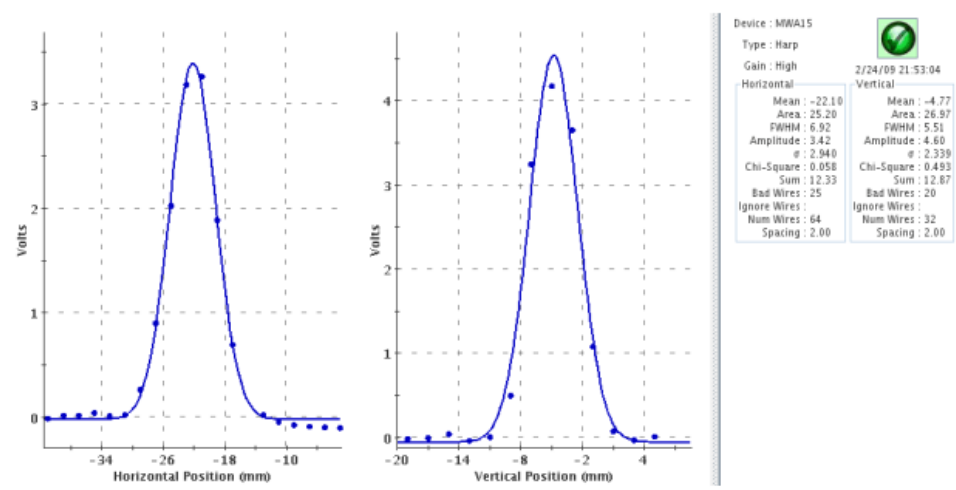

Multi-turn, no scraping: $\mathrm{H}$ sigma $=4.18 \mathrm{~mm}, \mathrm{~V}$ sigma $=3.243 \mathrm{~mm}$ (with single gaussian fit)
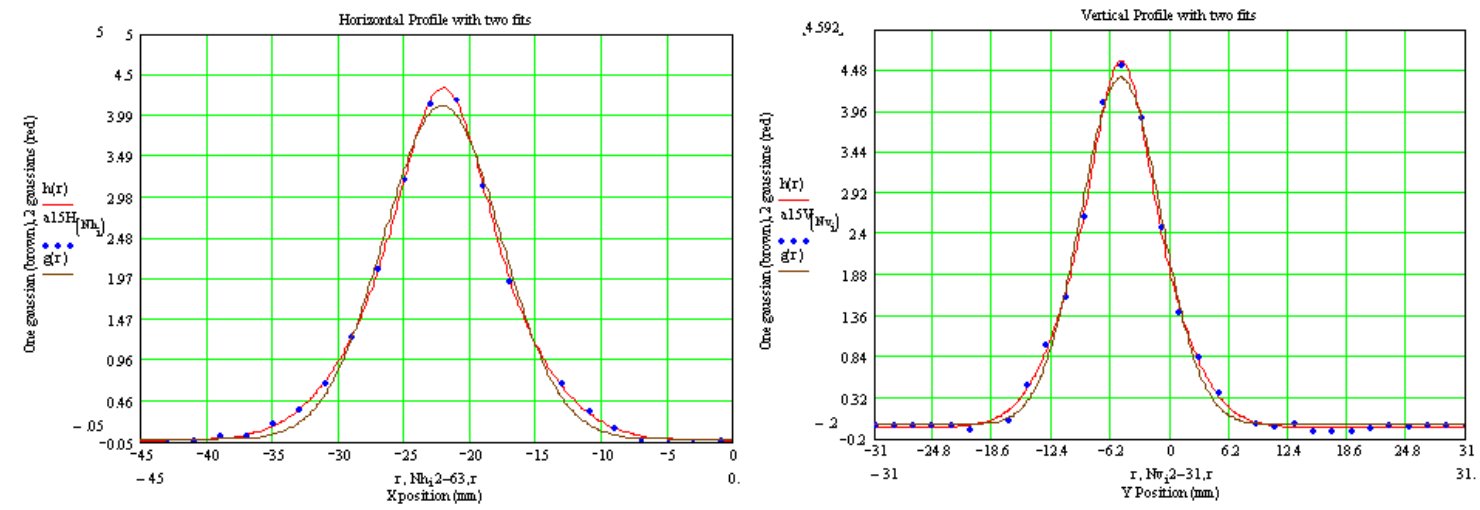

Figure 8: Single and multi-turn A15 Multiwire data (blue dots) for horizontal (left) and vertical (right) profiles. Single turn profiles have only single gaussian fits, and multi-turn profiles show both the single gaussian (brown) and the sum of two gaussians (red) fits (from 24 Feb 09, at 21:53 and 21:55, respectively).

\begin{tabular}{|l|l|l|}
\hline Case & Horizontal & Vertical \\
\hline Single turn $\sigma\left(\sigma_{\text {singleturn }}\right)$ & $2.94 \mathrm{~mm}$ & $2.34 \mathrm{~mm}$ \\
\hline Single Gaussian fit, multi-turn $\sigma\left(\sigma_{\mathrm{s}}\right)$ & $4.18 \mathrm{~mm}$ & $3.24 \mathrm{~mm}$ \\
\hline Double Gaussian fit, narrow Gaussian for multi-turn $\sigma\left(\sigma_{\mathrm{n}}\right)$ & $2.67 \mathrm{~mm}$ & $2.42 \mathrm{~mm}$ \\
\hline$\left(\sigma_{\mathrm{s}} / \sigma_{\text {singleturn }}\right)$ for single gaussian fit & 1.41 & 1.38 \\
\hline$\left(\sigma_{\mathrm{n}} / \sigma_{\text {singleturn }}\right)$ for double gaussian fit & 0.89 & 1.05 \\
\hline Narrow Gaussian Area/Wide Gaussian Area & 0.31 & 0.23 \\
\hline
\end{tabular}

Table VIII: Gaussian fit $\sigma$ values $\sigma_{\text {singleturn }}, \sigma_{\mathrm{s}}, \sigma_{\mathrm{n}}$, as well as $\sigma_{\mathrm{n}} / \sigma_{\text {singleturn }}$, and $\sigma_{\mathrm{s}} / \sigma_{\text {singleturn }}$, and the ratio of narrow to wide gaussian areas for the A15 multiwire profiles in figure 8. 
Figure 9 shows the same multi-turn data for unscraped beam as in figure 8, but this time besides the sum of the two gaussians, the individual narrow and wide gaussians are also shown. Unlike in the scraped beam case considered above (fig. 7), the wider gaussians have more area than the narrow gaussians.
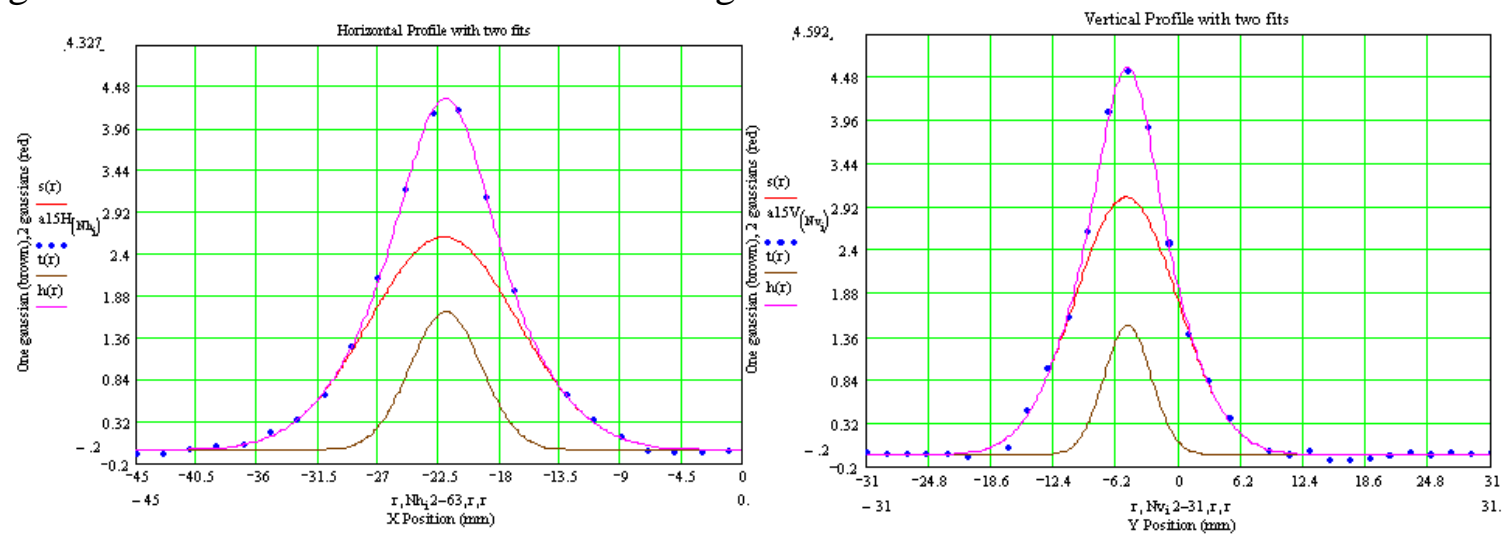

Figure 9: Horizontal (left) and vertical (right) A15 Multiwire data (blue dots) and fits to the sum of two gaussians (magenta curves) for the unscraped multi-turn profiles in figure 8 . The two gaussians themselves are also shown (red and brown curves).

Two other cases of single and multi-turn data are shown below in figures 10, 11, and 12, and in table IX. These two sets of data are from beam that has been scraped in the Booster in both planes. Figure 11 does show, as with the first case (figure 6) that the tails are more pronounced than in the unscraped case. Table IX shows that the horizontal $\left(\sigma_{\mathrm{n}} / \sigma_{\text {singleturn }}\right)$ is near 1 in the Feb $27^{\text {th }}$ case and considerably less than 1 in the Mar $3^{\text {rd }}$ case, and that the vertical $\left(\sigma_{\mathrm{n}} / \sigma_{\text {singleturn }}\right)$ is greater than 1 in both cases (1.37 and 1.26). There is not an obvious pattern in the relative areas of the wide and narrow gaussians, but in each case the double gaussian fits are quite good and significantly better than the single gaussian fits (see figure 11).
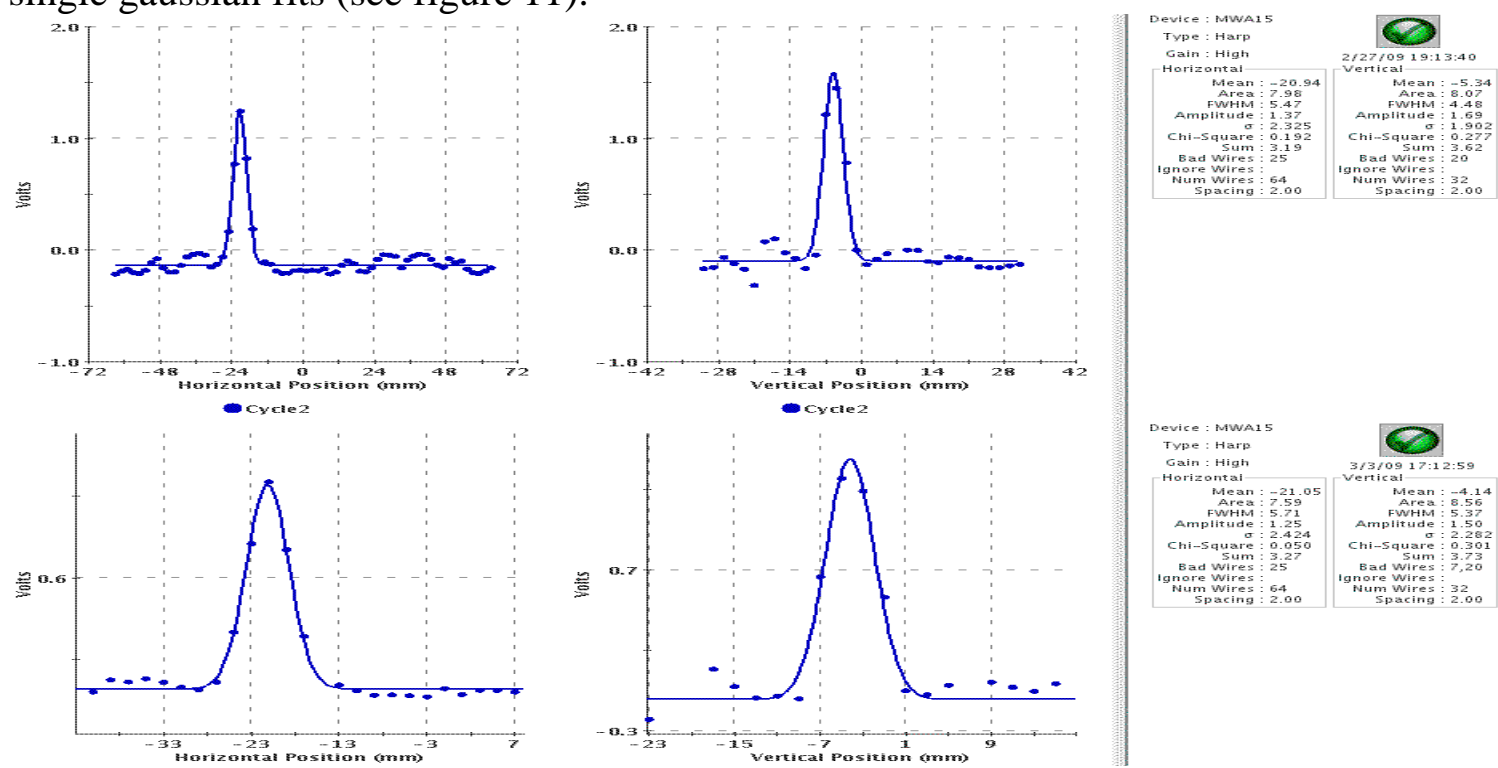

Figure 10: Horizontal (left) and vertical (right) A15 Multiwire data and gaussian fits for single turn profiles from Feb $27^{\text {th }}$ (top, scraped in both planes, profiles acquired at 19:13:40) and Mar $3^{\text {rd }}$ ( bottom, profiles acquired at 17:12:59). 

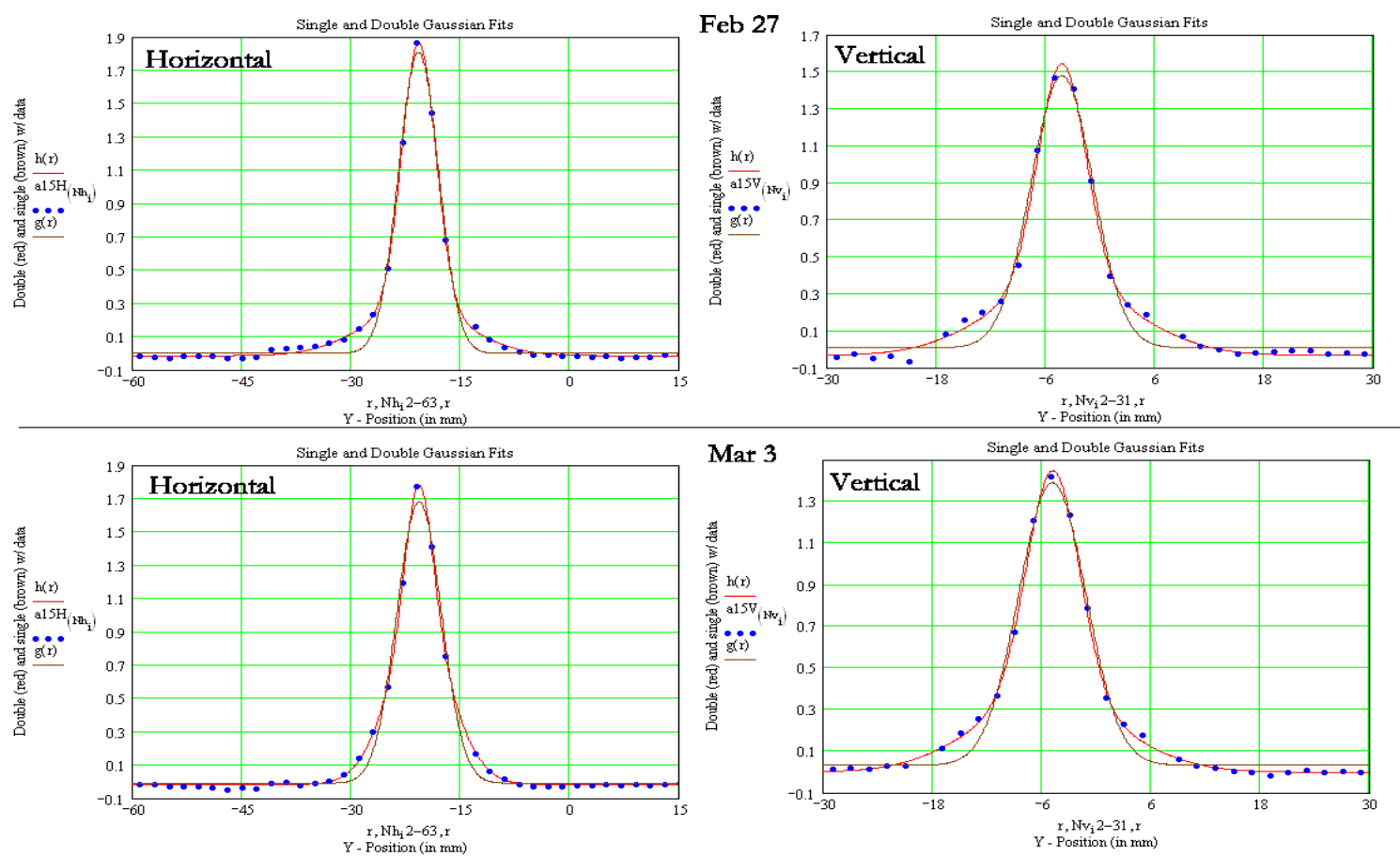

Figure 11: Multi-turn A15 Multiwire data (blue dots) for horizontal (left) and vertical (right) profiles for Feb 27 (top) and Mar 3 (bottom). Both the single gaussian (brown) and the sum of two gaussians (red) fits are shown.
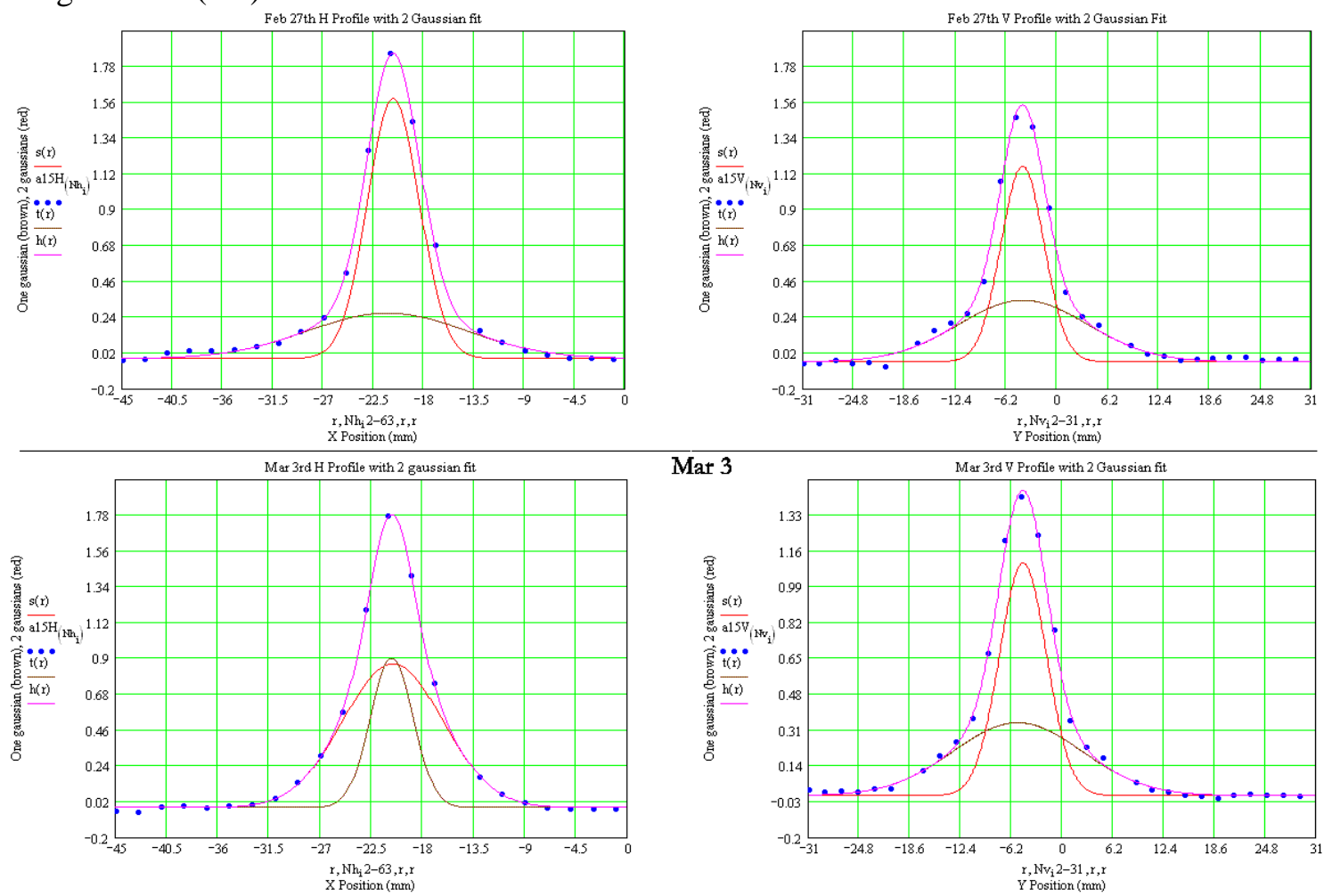

Figure 12: Horizontal (left) and vertical (right) A15 Multiwire data (blue dots) and fits to the sum of two gaussians (magenta curves) for multi-turn profiles from Feb $27^{\text {th }}$ (top, scraped in both planes, profiles acquired at 19:19:21) and Mar $3^{\text {rd }}$ (bottom, scraped in both planes, profiles acquired at 17:09:21), The wide and narrow gaussians are also shown (red and brown curves). 


\begin{tabular}{|l|l|l|}
\hline Feb $\mathbf{2 7}^{\text {th }}$ & Horizontal & Vertical \\
\hline Single turn $\sigma\left(\sigma_{\text {singleturn }}\right)$ & $2.33 \mathrm{~mm}$ & $1.90 \mathrm{~mm}$ \\
\hline Single Gaussian fit, multi-turn $\sigma\left(\sigma_{\mathrm{s}}\right)$ & $2.75 \mathrm{~mm}$ & $3.40 \mathrm{~mm}$ \\
\hline Double Gaussian fit, narrow Gaussian for multi-turn $\sigma\left(\sigma_{\mathrm{n}}\right)$ & $2.32 \mathrm{~mm}$ & $2.60 \mathrm{~mm}$ \\
\hline$\left(\sigma_{\mathrm{s}} / \sigma_{\text {singleturn }}\right)$ for single gaussian fit & 1.18 & 1.79 \\
\hline$\left(\sigma_{\mathrm{n}} / \sigma_{\text {singleturn }}\right)$ for double gaussian fit & 1.00 & 1.37 \\
\hline Narrow Gaussian Area/Wide Gaussian Area & 1.8 & 1.0 \\
\hline
\end{tabular}

\begin{tabular}{|l|l|l|}
\hline March $\mathbf{3}^{\text {rd }}$ & Horizontal & Vertical \\
\hline Single turn $\sigma\left(\sigma_{\text {singleturn }}\right)$ & $2.42 \mathrm{~mm}$ & $2.28 \mathrm{~mm}$ \\
\hline Single Gaussian fit, multi-turn $\sigma\left(\sigma_{\mathrm{s}}\right)$ & $2.69 \mathrm{~mm}$ & $3.54 \mathrm{~mm}$ \\
\hline Double Gaussian fit, narrow Gaussian for multi-turn $\sigma\left(\sigma_{\mathrm{n}}\right)$ & $1.94 \mathrm{~mm}$ & $2.87 \mathrm{~mm}$ \\
\hline$\left(\sigma_{\mathrm{s}} / \sigma_{\text {singleturn }}\right)$ for single gaussian fit & 1.11 & 1.55 \\
\hline$\left(\sigma_{\mathrm{n}} / \sigma_{\text {singleturn }}\right)$ for double gaussian fit & 0.80 & 1.26 \\
\hline Narrow Gaussian Area/Wide Gaussian Area & 0.50 & 1.14 \\
\hline
\end{tabular}

Table IX: Gaussian fit $\sigma$ values $\sigma_{\text {singleturn }}, \sigma_{\mathrm{s}}, \sigma_{\mathrm{n}}$, as well as $\sigma_{\mathrm{n}} / \sigma_{\text {singleturn }}$, and $\sigma_{\mathrm{s}} / \sigma_{\text {singleturn }}$, and the ratio of narrow to wide gaussian areas for the A15 multiwire profiles in figures 10-12.

\section{Why Do the Multi-turn Profiles have a Double Gaussian Shape?}

It seems that the reason why the sum of two gaussians does a better job of fitting the multi-turn data is related to the fact that the tails are disproportionately wider than the center of the distribution when compared to a single gaussian (in the single turn cases, a double gaussian fit contributes nothing). But what the reason is for this is not clear. It may be beam that has scattered on the multiwire interacting with it again on subsequent turns, or it might be due to some kind of injection mismatch. Additionally, if the shape were solely due to scattering on the multiwire, then the narrow gaussian would be representative of the beam unaffected by the multiwire, and the wider gaussian would be a result of the scattering.

In order to explore the possibility that the double gaussian shape may be due to an injection mismatch, figure 13 shows what a $\beta$ function mismatch would look like in the case of an incoming gaussian beam with $\alpha=\alpha_{0}=0$. In this case, one can see as the beam becomes more mismatched the tails become more pronounced, and the center becomes, if anything, more sharply peaked. On the other hand, a steering mismatch produces profiles where the center becomes less sharply peaked, and the tails are not so affected. ${ }^{19}$ Their shapes in that case are closer to those of two superimposed gaussians with different center positions. A dispersion mismatch also produces a wider center. ${ }^{20}$ A dispersion mismatch is rather unlikely in the vertical, since although the snakes, and other things, can introduce coupling, efforts were made during the run to minimize coupling, and little

\footnotetext{
${ }^{19}$ See figure 7.3 on pg. 229 of "An Introduction to the Physics of High Energy Accelerators", D.A. Edwards and M.J. Syphers, 1993.

${ }^{20}$ See figure 7.4 on pg. 232 of "An Introduction to the Physics of High Energy Accelerators", D.A.

Edwards and M.J. Syphers, 1993.
} 
coupling was observed with the tunemeter near injection. So, the two mechanisms that would seem to favor producing more pronounced tails without affecting the sharpness of the center much would seem to be scattering from the multiwire or an optical mismatch.

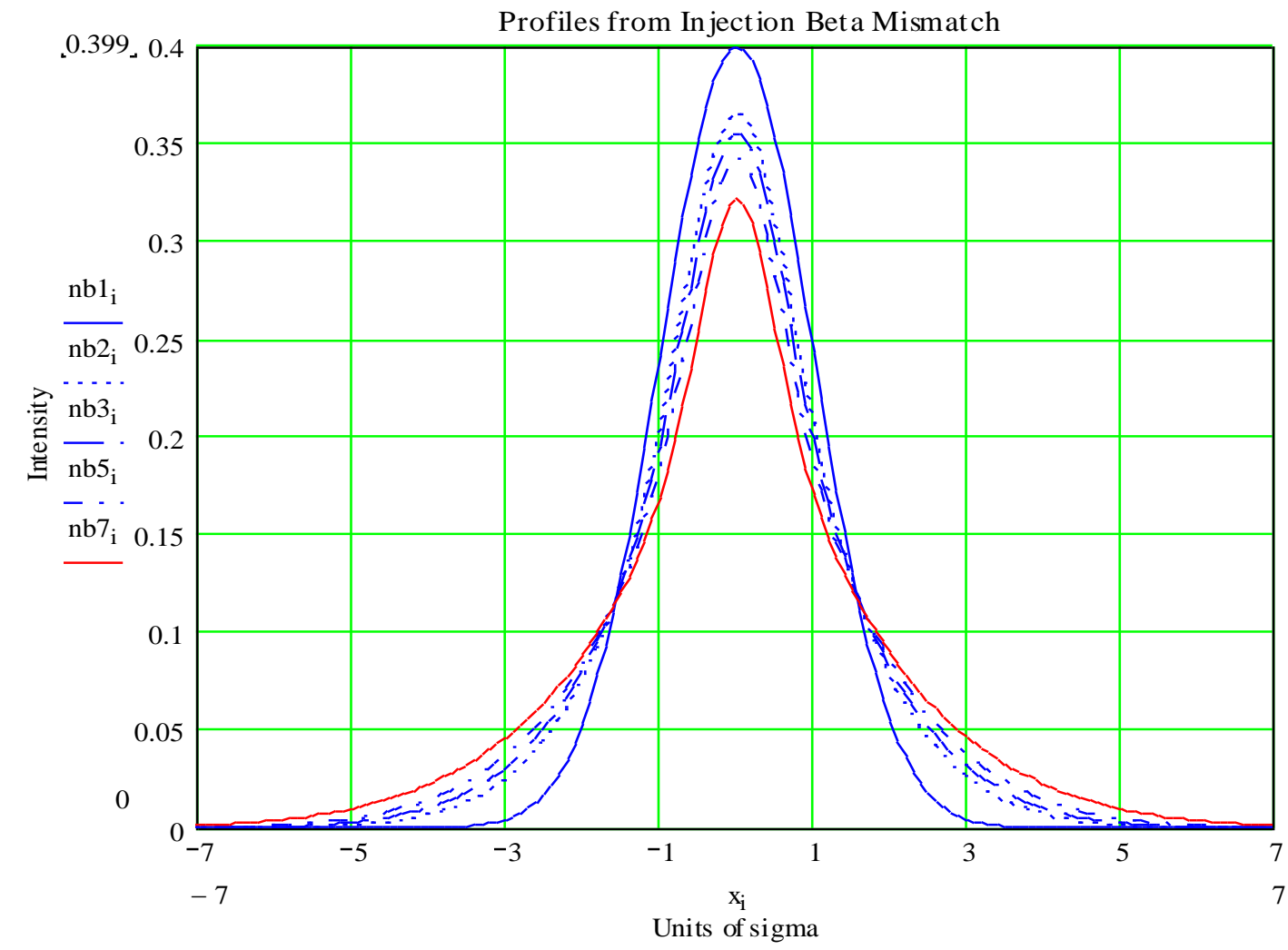

Figure 13: Particle Distributions due to different magnitudes of $\beta$ function mismatch at injection (for the special case where $\alpha=\alpha_{0}=0$ ). Solid Blue: No Mismatch, Dotted Blue: $\Delta \beta / \beta_{0}=1$, Dashed blue: $\Delta \beta / \beta_{\mathrm{o}}=-0.7$, Dash-dot blue: $\Delta \beta / \beta_{0}=-0.75$, and Red solid: $\Delta \beta / \beta_{0}=6$. Where $\Delta \beta=\beta-\beta_{\mathrm{o}}, \beta$ is the $\beta$ function of the incoming beam, and $\beta_{\mathrm{o}}$ is the lattice $\beta$ function. ${ }^{21}$

\section{A Double Gaussian Fits the Distribution from a $\beta$ Function Mismatch}

Figure 14 shows the profile for a $\beta$ function mismatch (with $\alpha=\alpha_{0}=0$ ) in black (where the incoming $\beta$ is three times the lattice $\beta$, or $\Delta \beta / \beta_{\mathrm{o}}=2$ where $\Delta \beta=\beta-\beta_{\mathrm{o}}$ ), a single gaussian fit to that data in blue, a double gaussian fit to that data in red, and the profile for the incoming beam in magenta. Even though the equation used to generate this distribution is not the sum of two gaussians, the double gaussian fit does a very good job of reproducing it. ${ }^{22}$ The $\sigma\left(=\sigma_{\mathrm{o}}\right)$ of the matched beam (not shown in the figure) is 1 , $\sigma_{\mathrm{s}}=1.34, \sigma_{\mathrm{n}}=0.70, \sigma_{\mathrm{w}}=1.48$, and the incoming $\sigma, \sigma_{\text {singleturn }}=1.73$. Figure 15 shows the two gaussians that comprise the fit shown in figure 14 together with the fit and data.

\footnotetext{
${ }^{21}$ Eq 7.48 on pg. 236 of "An Introduction to the Physics of High Energy Accelerators", D.A. Edwards and M.J. Syphers, 1993 is used to make this plot. This plot is also very similar to figure 7.6 in the preceding reference, which is also on pg. 236.

${ }^{22}$ Eq 7.48 on pg. 236 of "An Introduction to the Physics of High Energy Accelerators", D.A. Edwards and M.J. Syphers, 1993 is used to generate thall the distributions for a $\beta$ function mismatch considered here.
} 


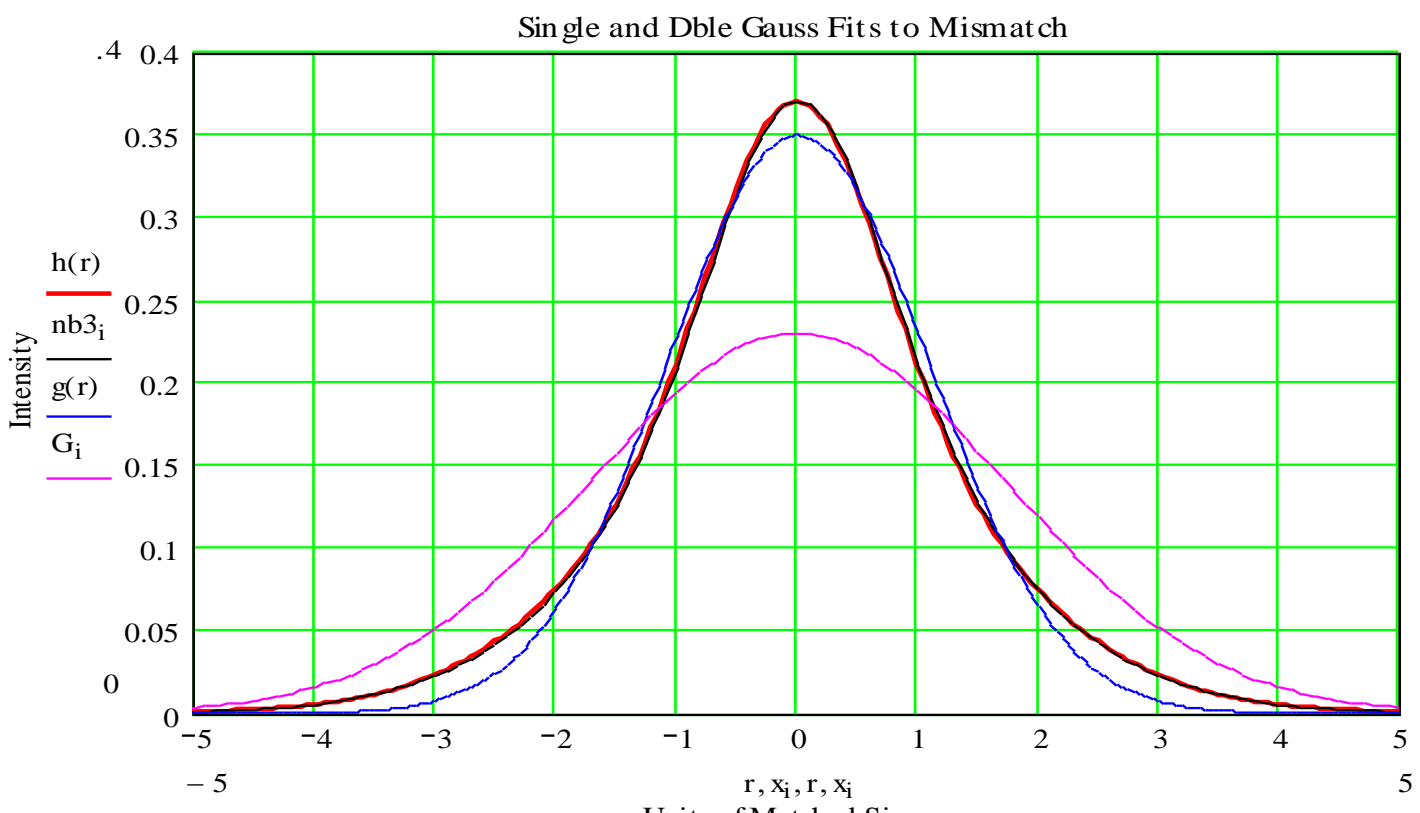

Units of Matched Sigma

Figure 14: Profile for a large $\beta$ function mismatch $\left(\Delta \beta / \beta_{o}=2\right.$, for the special case where $\left.\alpha=\alpha_{0}=0\right)$ in black, together with two fits. The blue trace is a single gaussian fit to the mismatch data, the red trace is a sum of two gaussian fit, and the magenta trace is the profile of the incoming beam.

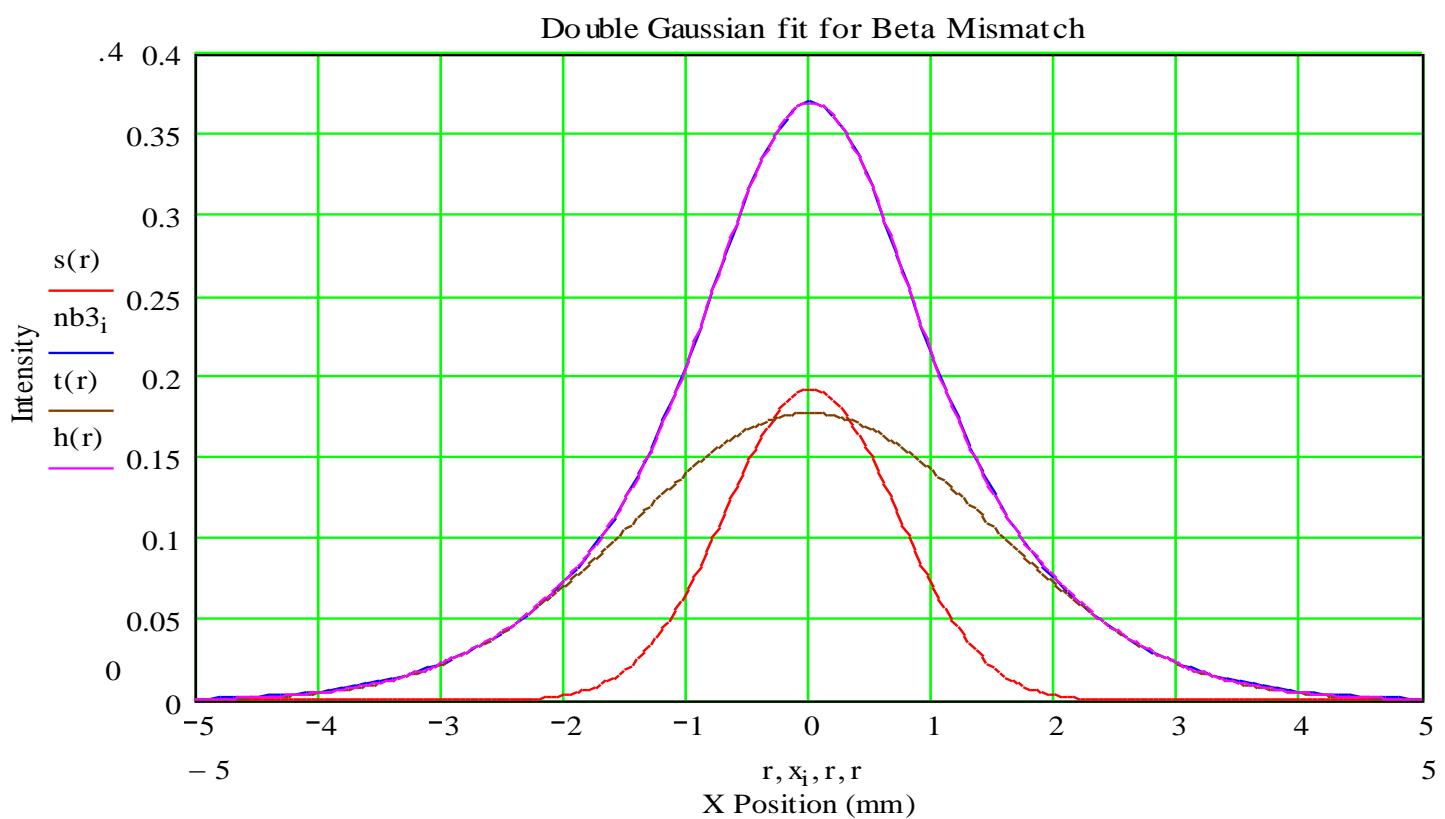

Figure 15: Profile for a large $\beta$ function mismatch $\left(\Delta \beta / \beta_{o}=2\right.$, for the special case where $\left.\alpha=\alpha_{o}=0\right)$ in blue, together with sum of two gaussian fit (magenta). The two gaussians that comprise the fit are also shown.

\section{Conflicting Indications}

In the case of a larger beam one might expect the effect of the scattering on the multiwire (the other possibility for the multi-turn shape) to be less obvious as the distributions of the scattered and unscattered beam would overlap more in the multi-turn 
profiles. If on the other hand, the larger tails were due to an $\beta$ mismatch (at least with $\alpha=\alpha_{0}=0$ ), the effect would be similar in either case as the shape of the resulting distribution is not a function of emittance. ${ }^{23}$ Figure 8 shows the case where the beam coming from the Booster has not been scraped, and is thereby larger than the beam in figure 6 (where it has been scraped). The tails on the multi-turn profiles in the unscraped case are not as pronounced as in the case with the scraped beam. Though not conclusive, this supports the idea that the change in profile shape between single and multi-turn in the scraped and unscraped cases is due to scattering on the multiwire, not an optical mismatch.

In the case of the unscraped beam the horizontal $\sigma_{\mathrm{n}}$ is even smaller than $\sigma_{\text {singleturn }}($ table VIII). This should not be the case if the change in shape were due solely to interaction with the multiwire, as the narrow gaussian should look just like the single turn gaussian in the case of unscraped beam. So, the fact that the horizontal narrow gaussian is narrower than the single turn case may be a sign of a $\beta$ function mismatch. As mentioned previously, this kind of behavior would not occur for a steering or dispersion mismatch as both these types of mismatch make the center of the distribution less sharp. Unlike in the scraped case, a comparison of the vertical $\sigma_{\mathrm{n}}$ with $\sigma_{\text {singleturn }}$ shows little difference.

\section{Connecting Different $\sigma$ Values in a $\beta$ Mismatch}

For the four sets of A15 multiwire data considered, the ratio $\left(\sigma_{\mathrm{n}} / \sigma_{\text {singleturn }}\right)$ in the horizontal ranges from 0.80 to 1.0 , and in the vertical it ranges from 1.05 to 1.37 .

The shape of the distribution resulting solely from a $\beta$ mismatch does not depend on the emittance of the incoming beam (at least with $\alpha=\alpha_{0}=0$ ), so it is possible to quantify how much of a mismatch in $\beta$ is required to produce the observed difference between the single turn and the multi-turn for the A15 multiwire data. This assumes that double gaussians fit the data well and there is a unique relationship between the narrow gaussian width and a given $\Delta \beta / \beta_{\mathrm{o}}$. This will be discussed further below.

To illustrate, by using eq 7.6 in Edwards and Syphers (see footnote 22) with a $\Delta \beta / \beta_{\mathrm{o}}$ of 0.30 a distribution is obtained. This distribution is fit to the sum of two gaussians as in figure 14, giving a value for the narrow gaussian $\sigma, \sigma_{\mathrm{n}}{ }^{\text {mismatch }}$, of $0.912 \sigma_{\mathrm{o}}$. Since the incoming $\beta$ is $1.30 \beta_{0}, \sigma_{\text {singleturn }}$ is $\sqrt{1.30 \beta_{o} \varepsilon_{o}}=1.14 \sigma_{o}$ (where $\sigma_{0}$ is the matched $\sigma$, and $\varepsilon_{0}$ is the incoming emittance). This makes the ratio $\sigma_{\mathrm{n}}{ }^{\text {mismatch }} / \sigma_{\text {singleturn }}$ equal to $0.912 \sigma_{\mathrm{o}} / 1.14 \sigma_{\mathrm{o}}=0.80$, and so, equating $\sigma_{\mathrm{n}}$ and $\sigma_{\mathrm{n}}{ }^{\text {mismatch }}$, this would be the $\beta$ mismatch for the horizontal Mar $3^{\text {rd }}$ data (again this is using the special case where $\alpha=\alpha_{0}=0$, as is the case in what follows as well).

\footnotetext{
${ }^{23}$ In "An Introduction to the Physics of High Energy Accelerators", pg 237, D.A. Edwards and M.J. Syphers, 1993 figure 7.6 shows that the distribution resulting from an optical mismatch doesn't change its shape as the $\sigma$ of the matched beam changes.
} 
Similarly, a value of 1.37 for $\sigma_{\mathrm{n}}{ }^{\text {mismatch }} / \sigma_{\text {singleturn, the maximum amount observed }}$ in the vertical for $\sigma_{\mathrm{n}} / \sigma_{\text {singleturn }}$ (Feb $27^{\text {th }}$ data), corresponds to a $\Delta \beta / \beta_{\mathrm{o}}$ of -0.80 , so that the incoming $\beta$ would be only $20 \%$ of the matched $\beta$. The measured $\sigma_{\text {singleturn }}$ would be $\sqrt{0.2} \sigma_{o}=0.45 \sigma_{o}$, and $\sigma_{\mathrm{n}}$, which is equated with $\sigma_{\mathrm{n}}{ }^{\text {mismatch }}$, would be $0.62 \sigma_{\mathrm{o}}$.

\section{Fitting Multiwire Data to a $\beta$ Mismatch Distribution}

This technique for finding the corresponding $\Delta \beta / \beta_{\mathrm{o}}$ for a given $\sigma_{\mathrm{n}} / \sigma_{\text {singleturn value }}$ can be used to compare A15 multiwire multi-turn data to a corresponding $\beta$ mismatch distribution. For example, figure 16 shows the feb $27^{\text {th }}$ vertical data together with the same double gaussian fit shown in figure 11 as well as a $\beta$ mismatch distribution (using eq. 7.48 in Edwards and Syphers) with the characteristics described above that give $\sigma_{\mathrm{n}}{ }^{\text {mismatch }} / \sigma_{\text {singleturn }}=1.37$. Specifically, to give a $\sigma_{\mathrm{n}}{ }^{\text {mismatch }}$ of 2.60 (as in table IX), the position values are multiplied by $2.60 / 0.616$ (where 0.616 is the width of the narrow gaussian described above that gives $\sigma_{\mathrm{n}}{ }^{\text {mismatch }} / \sigma_{\text {singleturn }}=1.37$ ). The same position offset that the double gaussian fit has is applied to the $\beta$ mismatch curve, and the intensity values of the $\beta$ mismatch distribution are multiplied by the ratio of the peak of the A15 data fit over the peak of the $\beta$ mismatch peak $(1.56 / 0.343=4.61)$ to make the peaks of the two curves the same.

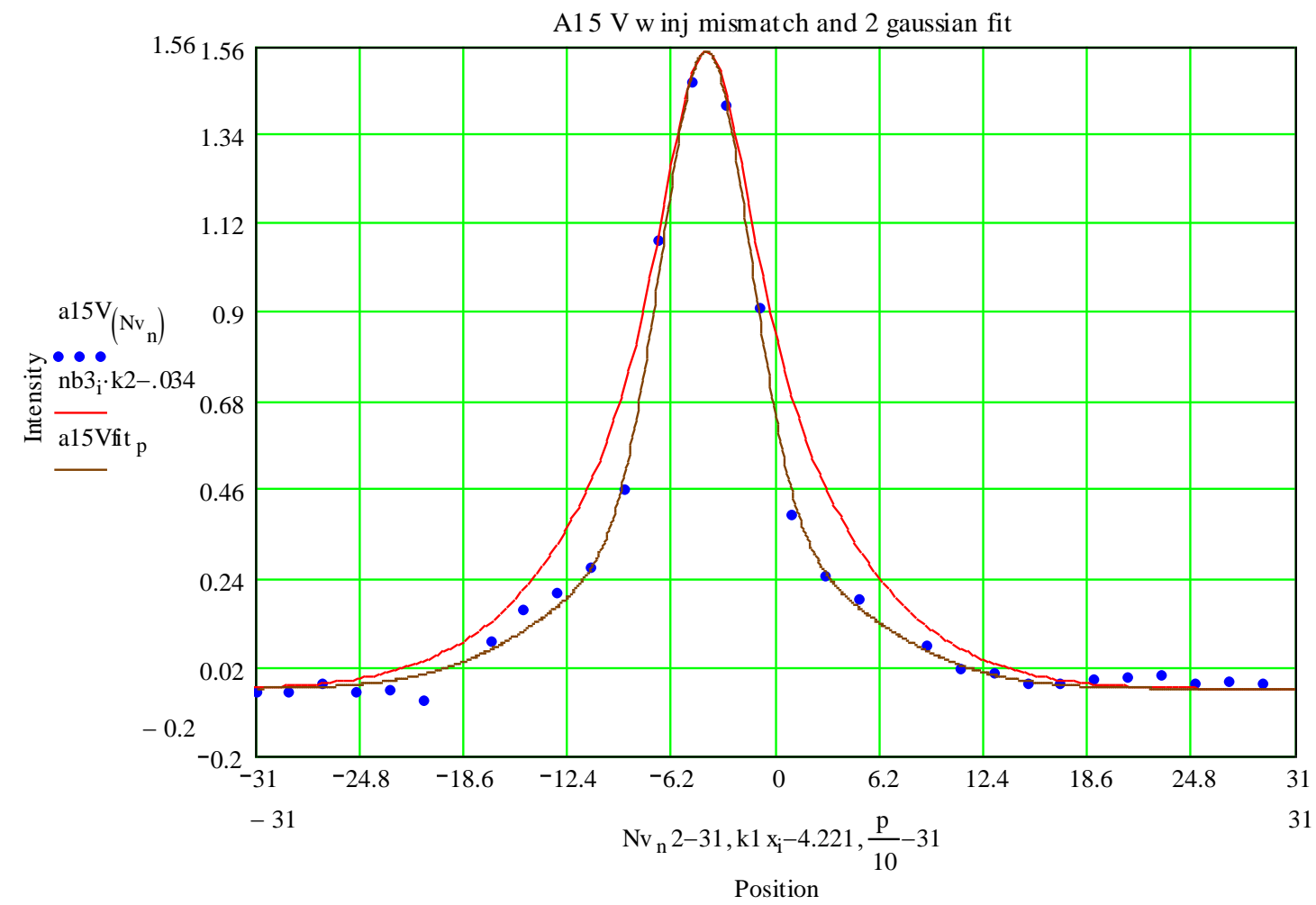

Figure 16: A15 Vertical Multi-turn Feb $27^{\text {th }}$ data (same as in fig. 15-blue dots) together with double gaussian fit (brown, same as in fig 11), and $\beta$ mismatch 'fit' (red-for the special case where $\alpha=\alpha_{0}=0$ ). 
The figure shows that the double gaussian fit obtained by fitting the A15 multiwire data is much better than the $\beta$ mismatch 'fit'. One might think that if the mechanism responsible for the shape of the profiles was a $\beta$ mismatch that the mismatch 'fit' using this method would work well. So, although a double gaussian fit to the distribution resulting from a $\beta$ mismatch works well, it is not the same double gaussian that best fits the multiwire data. Suggesting that, at least in part the shape of the multiwire data is not due to a $\beta$ mismatch.

Figure 17 is similar to figure 16 , except that it is for the Mar $3^{\text {rd }}$ horizontal multiturn data discussed above where $\sigma_{\mathrm{n}} / \sigma_{\text {singleturn }}=0.80$. Once again the mismatch 'fit' is very different from the double gaussian fit to the A15 data. This time it is too narrow, instead of too wide.

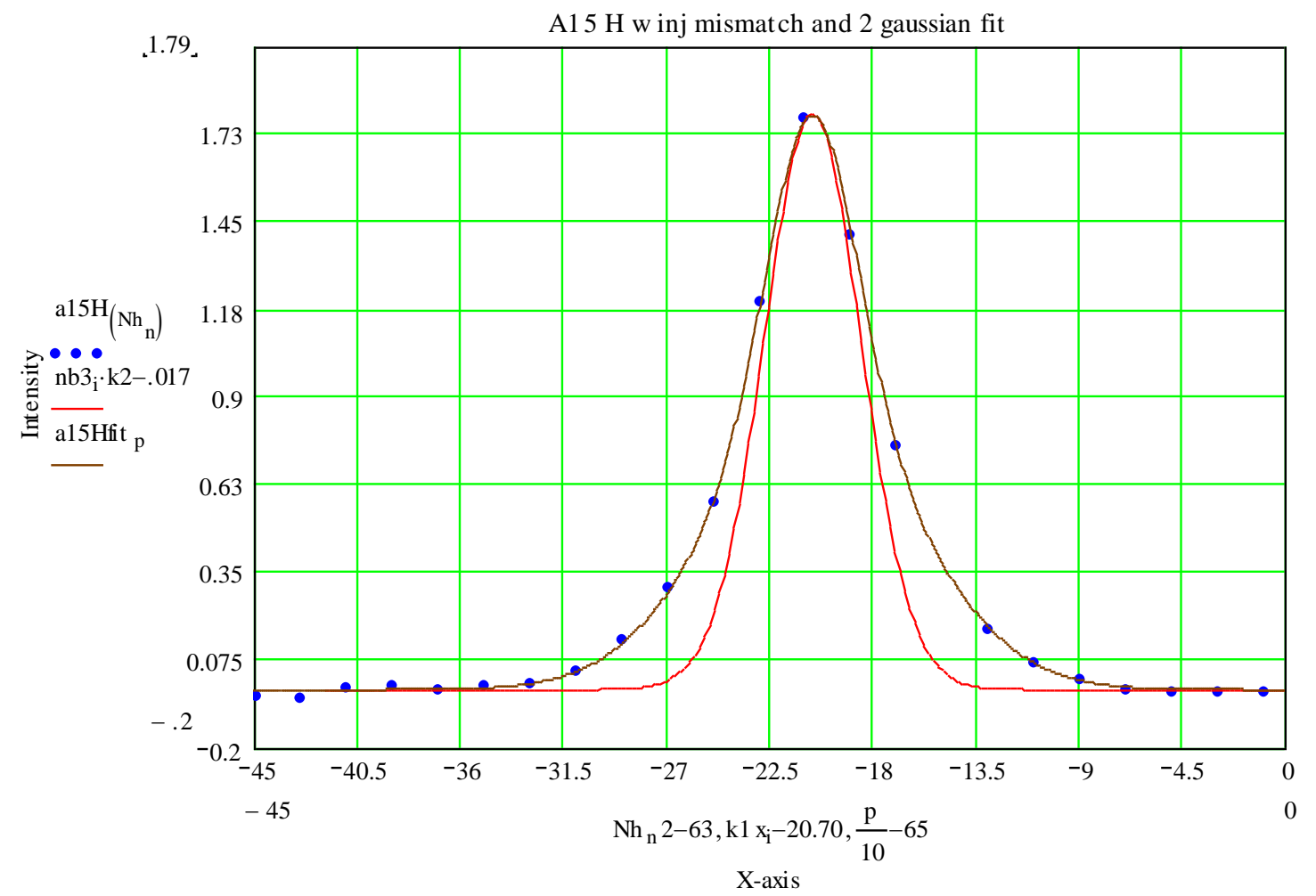

Figure 17: A15 Horizontal Multi-turn Mar $3^{\text {rd }}$ data (same as in fig. 15-blue dots) together with double gaussian fit (brown, same as in fig 11), and $\beta$ mismatch 'fit' (red-for the special case where $\alpha=\alpha_{0}=0$ ).

\section{$\sigma$ Values as Functions of $\Delta \beta / \beta_{o}$}

One might ask whether it is valid to associate a particular narrow gaussian $\sigma, \sigma_{\mathrm{n}}{ }^{\text {mismatch }}$, in the double gaussian fit of the mismatch distribution with a particular $\Delta \beta / \beta_{\mathrm{o}}$ value (for $\sigma_{\mathrm{o}}=1$ ). If, for $\sigma_{\mathrm{o}}=1$, there is a double gaussian fit that fits each $\beta$ mismatch distribution well, and there is only 1 value for $\sigma_{\mathrm{n}}{ }^{\text {mismatch }}$ for every value of $\Delta \beta / \beta_{\text {o }}$ then that would seem reasonable. 
By going through this process for different $\beta$ mismatches one can see the dependence of the $\sigma_{n}{ }^{\text {mismatch }}$ on $\Delta \beta / \beta_{o}$ and determine whether this is so (recall however that all of this is for the special case where $\alpha=\alpha_{0}=0$ ). Figure 18 shows $\sigma_{\mathrm{n}}{ }^{\text {mismatch }}$ vs. $\Delta \beta / \beta_{\mathrm{o}}$ in black (where $\sigma_{\mathrm{n}}{ }^{\text {mismatch }}$ is in units of $\sigma_{\mathrm{o}}$ ). It shows that $\sigma_{\mathrm{n}}{ }^{\text {mismatch }}$ never exceeds $\sigma_{\mathrm{o}}(=1$ ), is only equal to $\sigma_{\mathrm{o}}$ in the matched case, and in particular has a single value for each $\Delta \beta / \beta_{\mathrm{o}}$ over the range considered. Additionally, a scan of double gaussian fits vs $\Delta \beta / \beta_{\mathrm{o}}$ values shows that the fits are generally good (as in fig 14). So, though this is far from a rigorous proof, the condition mentioned above appears to be met reasonably well. Other $\sigma$ values are also shown. The $\sigma_{\mathrm{w}}$ mismatch (magenta) is never less than $\sigma_{\mathrm{o}}$ and is nonexistent when the beam is matched, the $\sigma_{\mathrm{s}}^{\text {mismatch }}$ (red) is never less than $\sigma_{0}$, and the $\sigma_{\text {singleturn }}$ of the incoming beam (blue) is less than $\sigma_{\mathrm{o}}$ when $\Delta \beta / \beta_{\mathrm{o}}<1$ and greater than $\sigma_{\mathrm{o}}$ when $\Delta \beta / \beta_{0}>1$.

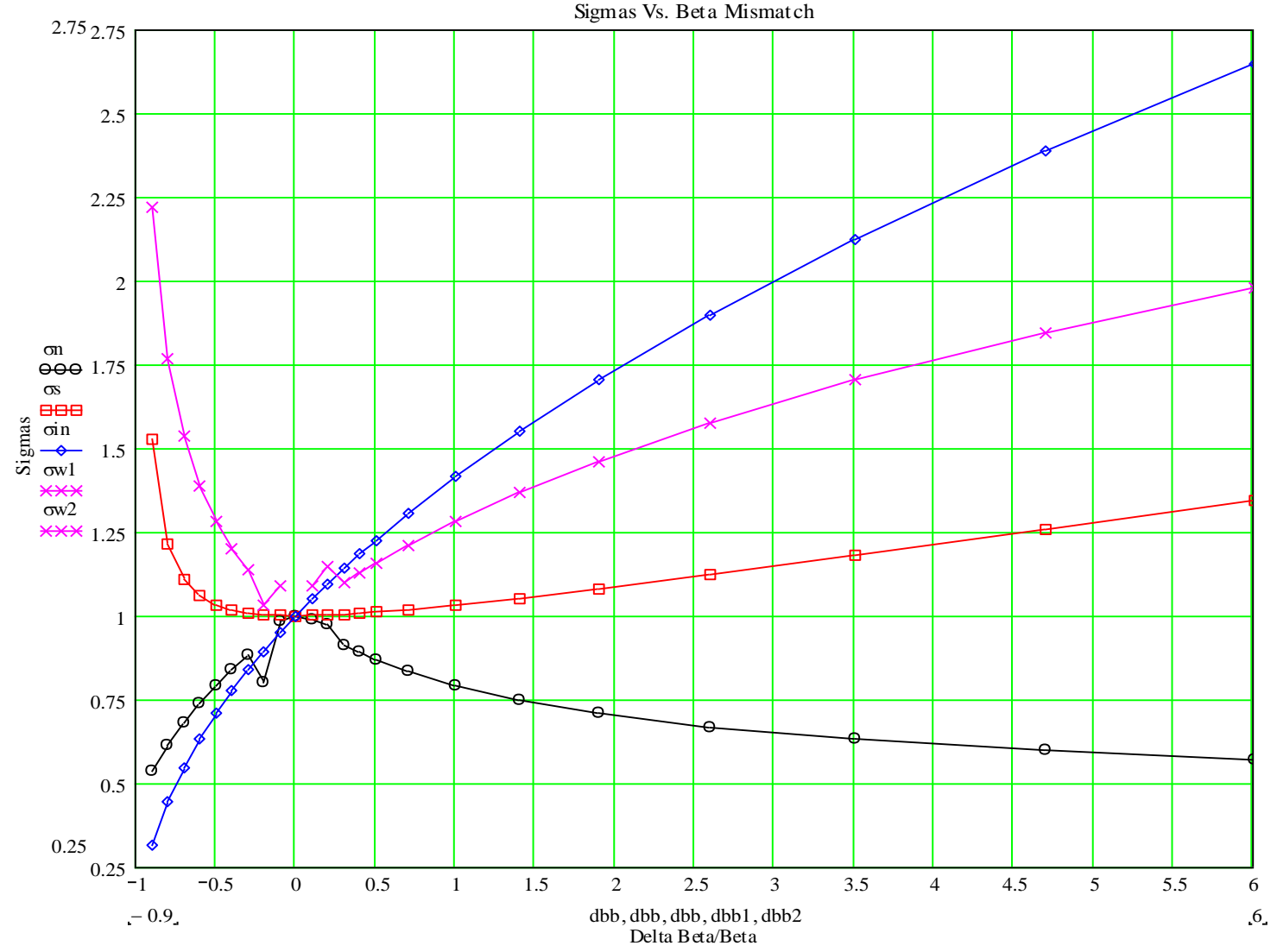

Figure 18: $\Delta \beta / \beta_{\mathrm{o}}$ (x-axis) vs. $\sigma$ values in units of matched $\sigma$ (i.e. $-\sigma_{\circ}$ ) for the special case where $\alpha=\alpha_{0}=0$. The blue curve is the $\sigma$ of incoming beam $\left(\sigma_{\text {singleturn }}\right)$, the red is $\sigma$ for a single gaussian fit

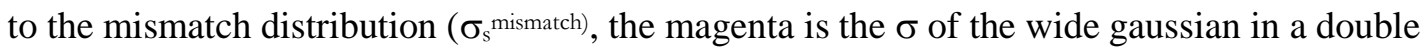
gaussian fit to the mismatch distribution $\left(\sigma_{\mathrm{w}}\right.$ mismatch), and the black is the $\sigma$ of the narrow gaussian in a double gaussian fit to the mismatch distribution $\left(\sigma_{\mathrm{n}}\right.$ mismatch $)$.

Figure 18 may be useful in making sense of data from the A15 multiwire. The ratios $\sigma_{\mathrm{n}}{ }^{\text {mismatch }} / \sigma_{\text {singleturn }}$ and $\sigma_{\mathrm{s}}{ }^{\text {mismatch }} / \sigma_{\text {singleturn }}$ can be obtained from it. These ratios can be plotted against $\Delta \beta / \beta_{\mathrm{o}}$ (as in figure 19), and assuming that the changes in the multi-turn profiles are due to a $\beta$ mismatch, the values for $\Delta \beta / \beta_{\text {o }}$ can be determined for each plane 
and in each of the four cases (i.e.- assuming $\sigma_{\mathrm{n}}=\sigma_{\mathrm{n}}{ }^{\text {mismatch }}$ and $/$ or $\sigma_{\mathrm{s}}=\sigma_{\mathrm{s}}{ }^{\text {mismatch }}$ ). Table $\mathrm{X}$ shows the results. Assuming the mismatch is about the same in all four cases, one might expect the values for $\Delta \beta / \beta_{\mathrm{o}}$ to be similar as well over the span of the four cases, and using either method. Averaging over the four cases, the horizontal $\Delta \beta / \beta_{\mathrm{o}}$ would then be $-0.31^{+}-0.11$ if $\sigma_{\mathrm{s}}=\sigma_{\mathrm{s}}{ }^{\text {mismatch }}$ and $+0.12^{+}{ }_{-} 0.13$ if $\sigma_{\mathrm{n}}$ is taken as $\sigma_{\mathrm{n}}{ }^{\text {mismatch }}$. The vertical $\Delta \beta / \beta_{\mathrm{o}}$ is in better agreement, $-0.53^{+} 0.07$ for $\sigma_{\mathrm{s}}=\sigma_{\mathrm{s}}{ }^{\text {mismatch }}$ and $-0.58^{+}-0.23$ for $\sigma_{\mathrm{n}}=\sigma_{\mathrm{n}}{ }^{\text {mismatch }}$.

The expected emittance blowup for a $\beta$ mismatch can be found using,

$$
\frac{\sigma^{2}}{\sigma_{o}^{2}}=1+\frac{1}{2}\left(\frac{\Delta \beta / \beta_{o}}{\sqrt{1+\Delta \beta / \beta_{o}}}\right)^{2}
$$

from Edwards and Syphers for the special case where $\alpha=\alpha_{0}=0$ (where $\sigma$ is the for the resulting distribution and $\sigma_{\mathrm{o}}$ is for the matched case). ${ }^{24}$ This is also plotted in black in figure 19 . From this equation and the values for $\Delta \beta / \beta_{\mathrm{o}}$ obtained above, one can determine the emittance growth one would expect using these two methods for each of the four cases. The last row in table $\mathrm{X}$ shows the results. In the horizontal one would expect the emittance to increase by less than $10 \%$ and in the vertical to increase between 30 and $40 \%$. But if error bars on $\Delta \beta / \beta_{\mathrm{o}}$ are considered, the horizontal could grow by as much as $16 \%$ and the vertical by as much as $185 \%$ (2.85 times) or by as little as $9 \%$. Still, even if the change in beam size seen on the A15 multiwire between the single and multi-turn cases could be attributed to a $\beta$ mismatch, the associated increase in emittance is likely not enough to account for what is observed with the IPM on the AGS flattop, at least in the vertical, where the increase ranges from about 2.5-3 times with unscraped beam to 13-14 times with heavily scraped beam (figure 4).

\begin{tabular}{|c|c|c|c|c|}
\hline & $\begin{array}{l}\mathbf{H} \\
\sigma_{\mathrm{s}}{ }_{\text {mismatch }} / \sigma_{\text {singleturn }} \\
\Delta \beta / \beta_{\mathrm{o}}\end{array}$ & $\begin{array}{l}\mathbf{H} \\
\sigma_{\mathrm{n}}{ }_{\text {mismatch }} / \sigma_{\text {singleturn }} \\
\Delta \beta / \beta_{\mathrm{o}}\end{array}$ & $\begin{array}{l}\mathbf{V} \\
\sigma_{\mathrm{s}}{ }^{\text {mismatch }} / \sigma_{\text {singleturn }} \\
\Delta \beta / \beta_{\mathrm{o}}\end{array}$ & $\begin{array}{l}\mathrm{V} \\
\sigma_{\mathrm{n}}{ }^{\text {mismatch }} / \sigma_{\text {singleturn }} \\
\Delta \beta / \beta_{\mathrm{o}}\end{array}$ \\
\hline Feb 23 & -0.32 & 0 & -0.51 & -0.68 \\
\hline Feb 24 & -0.47 & +0.20 & -0.45 & -0.21 \\
\hline Feb 27 & -0.27 & 0 & -0.63 & -0.80 \\
\hline Mar 3 & -0.18 & +0.30 & -0.52 & -0.64 \\
\hline $\operatorname{Mean}^{+} \sigma \sigma$ & $-0.31^{+} .0 .11$ & $+0.12^{+}-0.13$ & $-0.53^{+} .0 .07$ & $-0.58^{+}-0.23$ \\
\hline $\begin{array}{l}\text { Emit. } \\
\text { growth }\end{array}$ & $\begin{array}{l}1.07(\min 1.03 \\
\max 1.16)\end{array}$ & $\begin{array}{l}1.01(\min 1.00, \\
\max 1.03)\end{array}$ & $\begin{array}{l}1.30(\min 1.21, \\
\max 1.45)\end{array}$ & $\begin{array}{l}1.40(\min 1.09, \\
\max 2.85)\end{array}$ \\
\hline
\end{tabular}

Table X: Expected $\Delta \beta / \beta_{\text {o }}$ for each A15 MW profile set using the relationships between $\sigma_{\mathrm{s}}{ }^{\text {mismatch }} / \sigma_{\text {singleturn }}$ or $\sigma_{\mathrm{n}}{ }^{\text {mismatch }} / \sigma_{\text {singleturn }}$ and $\Delta \beta / \beta_{\mathrm{o}}$ shown in figure 19 . The factor by which the emittance would be expected to increase, also indicated in figure 19 , is also included for the mean and plus or minus the standard deviation values for $\Delta \beta / \beta_{\mathrm{o}}$.

Alternately, the relationships shown in figure 19 could be plotted as in figure 20 where the $\mathrm{x}$-axis is the factor by which the emittance grows, and the $\mathrm{y}$-axis is either

\footnotetext{
${ }^{24}$ Eq 7.58 on pg. 237 of "An Introduction to the Physics of High Energy Accelerators”, pg 237, D.A. Edwards and M.J. Syphers, 1993.
} 


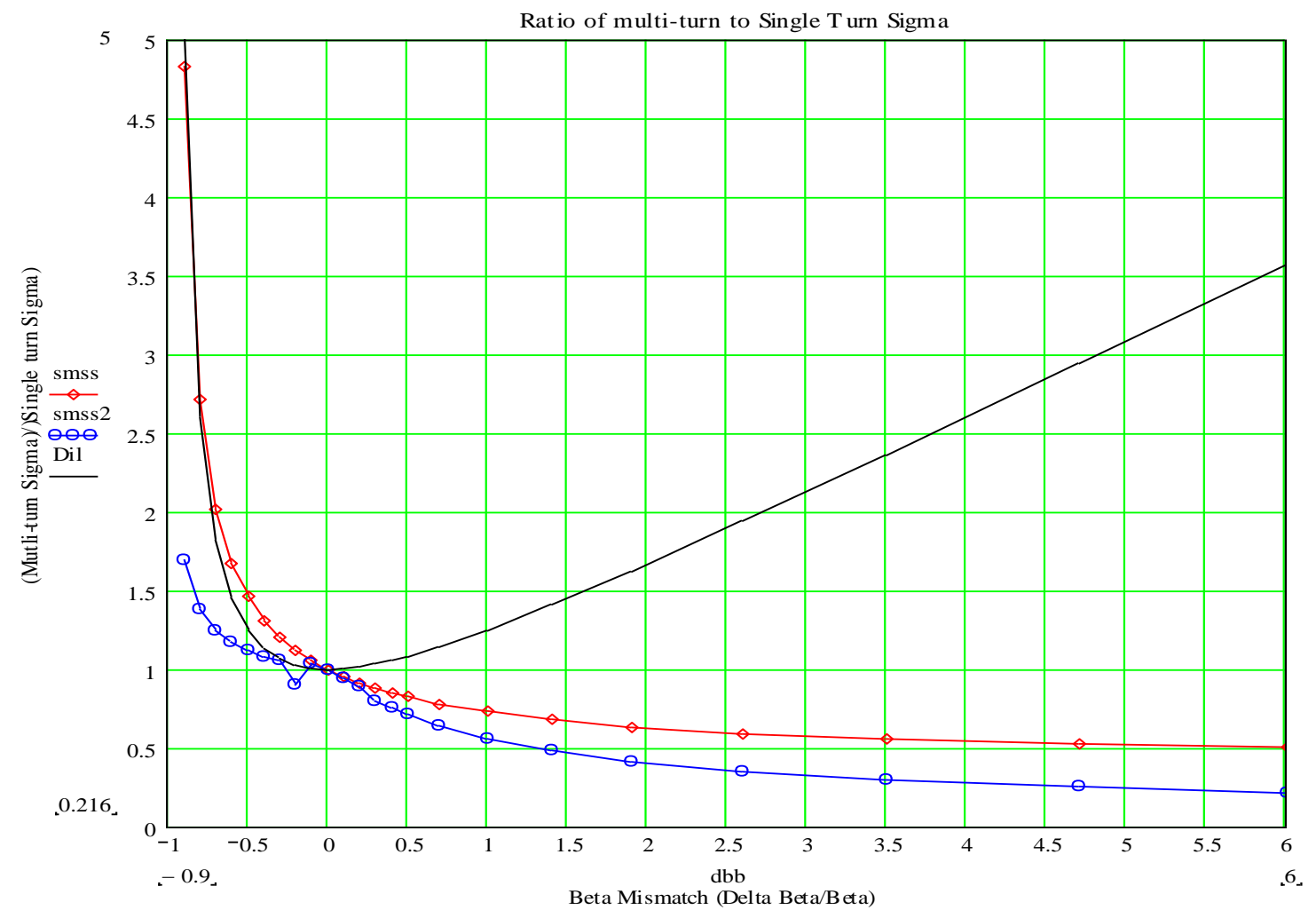

Figure 19: $\Delta \beta / \beta_{\mathrm{o}}$ (x-axis) vs. $\sigma_{\mathrm{n}}{ }^{\text {mismatch }} / \sigma_{\text {singleturn }}$ (blue) and $\sigma_{\mathrm{s}}{ }^{\text {mismatch }} / \sigma_{\text {singleturn }}(\mathrm{red})$, and $\left(\sigma / \sigma_{\mathrm{o}}\right)^{2}$ (black). For the special case where $\alpha=\alpha_{0}=0$.

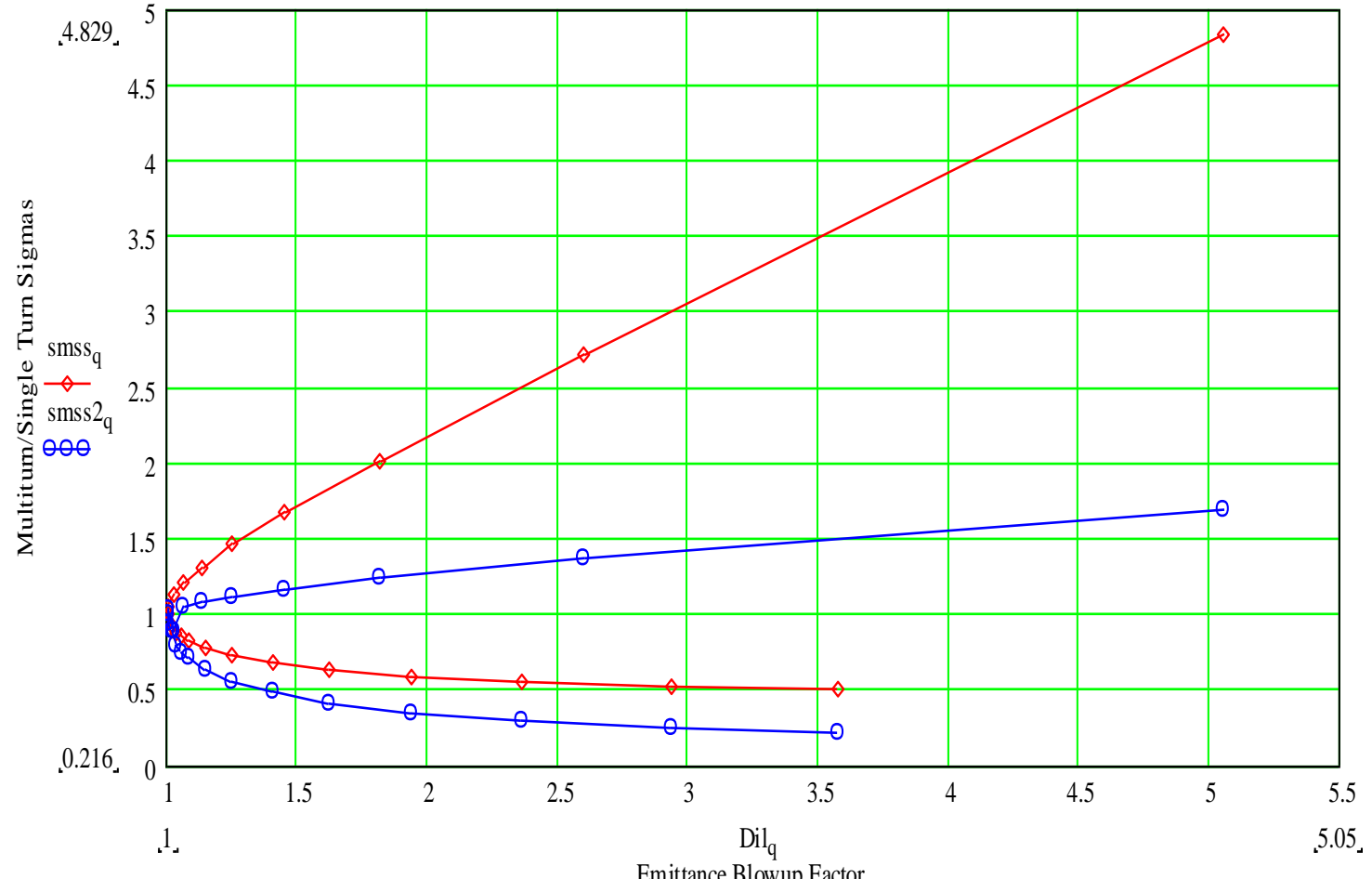

Figure 20: Emittance blowup factor vs. $\sigma_{\mathrm{n}}{ }^{\text {mismatch }} / \sigma_{\text {singleturn }}$ (blue) or ${\sigma_{\mathrm{s}}}^{\text {mismatch }} / \sigma_{\text {singleturn }}$ (red) for a $\beta$ mismatch with $\alpha=\alpha_{0}=0$. 
$\sigma_{\mathrm{n}}{ }^{\text {mismatch }} / \sigma_{\text {singleturn }}$ or $\sigma_{\mathrm{s}}{ }^{\text {mismatch }} / \sigma_{\text {singleturn. Equating } \sigma_{\mathrm{n}}}{ }^{\text {mismatch }}$ with $\sigma_{\mathrm{n}}$ and $\sigma_{\mathrm{s}}{ }^{\text {mismatch }}$ with $\sigma_{\mathrm{s}}$ allows one to determine how much emittance blowup to expect if the change in $\sigma_{\mathrm{n}}$ and $\sigma_{\mathrm{s}}$ from $\sigma_{\text {singleturn }}$ were due to a $\beta$ mismatch.

Unfortunately, though these relationships might be nice, it doesn't seem that the profile shapes on the A15 multiwire are primarily due to a $\beta$ mismatch since the fits shown in figures 16 and 17 are not in good agreeement with the data and the shape of the distribution changes as the incoming emittance changes (smaller tails with larger emittance). Regardless, these relationships suggest that even if the changes in profile shape were due to $\beta$ mismatches, those mismatches are not large enough to account for those observed with the AGS IPM on the flattop.

Admittedly, the situation is more complicated than this as only the special case where $\alpha=\alpha_{0}=0$ has been explored. A relationship exists for $\left(\sigma / \sigma_{0}\right)^{2}$ in terms of $\alpha, \alpha_{0}$, and $\Delta \beta / \beta_{\mathrm{o}}{ }^{25}$ but there is no basis for associating a mismatch when $\alpha$ and $\alpha_{\mathrm{o}}$ are not equal with values for $\sigma_{\mathrm{s}}{ }^{\text {mismatch }} / \sigma_{\text {singleturn }}$ or $\sigma_{\mathrm{n}}{ }^{\text {mismatch }} / \sigma_{\text {singleturn }}$ since profiles for these cases are not readily generated. Additionally, the large number of variables would make such an analysis unwieldly. It is clear however that values for $\alpha$ and $\alpha_{0}$, that are within apparently reasonable ranges, can have a significant effect on $\left(\sigma / \sigma_{\mathrm{o}}\right)^{2}$. So, this analysis is far from complete in that it does not consider these cases.

Though the decrease in the horizontal $\sigma_{\mathrm{n}}$ relative to $\sigma_{\text {singleturn }}$ in some cases seems likely due to a mismatch as it is hard to account for it in another way, the amount of blowup due to this may be quite small $(<10 \%)$. Whether the increase in $\sigma_{\mathrm{n}}$ relative to $\sigma_{\text {singleturn }}$ in the vertical is also indicative of a $\beta$ mismatch is harder to tell as other mechanisms, such as a steering mismatch, could also be responsible for it.

\section{Summary}

Reductions to the incoming Linac emittance prompted changes to the injection foil, injection scheme, and work on BtA matching, so that these reductions could be realized in the AGS and RHIC. Although there were significant gains in the emittance of the beam extracted from the Booster, there was not much of a difference measured in the AGS with the IPM on flattop, the typical way of measuring the emittance in the AGS. The emittance measured near injection in the AGS has better agreement with the BtA data, but is somewhat suspect due to uncertainty in the $\beta$ function at the IPM at injection energy with the snakes on.

The quantity $\varepsilon_{R M S}=\sqrt{\left(\varepsilon_{h}{ }^{2}+\varepsilon_{v}{ }^{2}\right) / 2}$, where $\varepsilon_{\mathrm{h}}$ and $\varepsilon_{\mathrm{v}}$ are the $95 \%$ normalized horizontal and vertical emittances, is used to quantify the reduction in emittance.

\footnotetext{
${ }^{25} \mathrm{Eq} 7.56$ on pg. 237 of Edwards and Syphers is the general equation for $\left(\sigma / \sigma_{\mathrm{o}}\right)^{2}$ in terms of the twiss parameters of the incoming beam and lattice. The 6 variables in that equation, $\alpha_{0}, \beta_{0}, \gamma_{0}, \alpha, \beta$, and $\gamma$, can be redueced to the 3 variables $\alpha_{\mathrm{o}}, \alpha$, and $\Delta \beta / \beta_{\mathrm{o}}$ by algebraic manipulation.
} 
Measurements at the Linac show that the Linac $\varepsilon_{R M S}$ has been reduced some $57 \%$ between FY'09 and FY'06. Whereas, with the use of the $100 \mu \mathrm{g} / \mathrm{cm}^{2}$ foil and $1 / 2$ integer injection, the $\varepsilon_{R M S}$ of the beam extracted from the Booster in FY'09 has been reduced about $40-42 \%$ from what it was in FY'06.

Part of this improvement is due to the new injection foils, particularly the strip foil. The strip foil, which provides close to $100 \%$, instead of about $85 \%$, stripping efficiency also provides beam that is considerably brighter than either the 100 or 200 $\mu \mathrm{g} / \mathrm{cm}^{2}$ standard foils (see table I). It, and the stamp foil, are clearly better choices than either of these foils. Half integer injection also seemed to reduce the emittances significantly (see table III). The $\varepsilon_{R M S}$ of unscraped beam at MW006 after $1 / 2$ integer injection was optimized was about $29 \%$ of what it was with the standard injection scheme.

A comparison of what the IPM reports for the AGS late emittance for roughly standard running conditions in both the FY'06 and FY'09 runs shows that although the BtA $\varepsilon_{R M S}$ was reduced by $33 \%$, the $\varepsilon_{R M S}$ measured on flattop by the IPM was only reduced by $11 \%$ between the two runs. Most of this reduction in BtA is apparent in the horizontal plane where $\varepsilon_{\mathrm{h}}$ goes from 14.8 to $9.7 \pi \mathrm{mm} \mathrm{mr}$, but $\varepsilon_{\mathrm{h}}$ only goes from 10.8 to $9.8 \pi \mathrm{mm}$ mr using the flattop IPM measurement. Additionally, the horizontal BtA emittance in FY'09 basically agrees with this IPM emittance measurement, but the

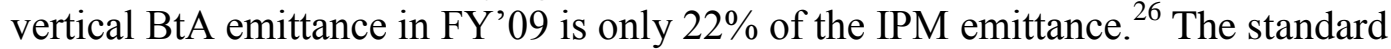
running FY'09 vertical BtA emittance (from table VI) is about the same as in FY'06, and the discrepancy between the IPM and BtA vertical emittances is similar in both runs. So, there are two issues: 1) Why isn't the horizontal BtA and IPM data from the two runs consistent? And, more glaringly, 2) Why is the vertical emittance measured in BtA 4 to 5 times smaller than measured with the IPM on flattop?

An optical mismatch at AGS injection is often thought to be at least partly responsible for the discrepancy between the BtA and AGS emittances. For most of the FY'09 run a new set of BtA optics was used that is based on a MAD model of the BtA line and the AGS, but IPM measurements with the new optics are not very different than those with the old optics. It has been observed, before the use of the new optics, and after as well, that multi-turn A15 multiwire profiles are usually wider than single turn profiles, particularly in the vertical plane.

For either plane, and whether the beam has been scraped or not, a better fit results if the multi-turn A15 multiwire data is fit to the sum of two gaussians instead of a single gaussian. One question is how to interpret this fact. If the narrower of the two gaussians is attributed to beam that has not significantly interacted with the foil, and the wider gaussian is attributed to beam that has scattered on the A15 multiwire wires, than the narrow gaussian should be more representative of the size of the injected beam in the AGS. So, its width can be compared to the single turn width for signs of an injection mismatch. A method was developed for determining the emittance growth due to a $\beta$

\footnotetext{
${ }^{26}$ See table VI
} 
mismatch (for the special case where $\alpha=\alpha_{0}=0$ ) given the narrow gaussian width, $\sigma_{\mathrm{n}}$, or the single fit gaussian width, $\sigma_{\mathrm{s}}$, and the single turn gaussian width $\sigma_{\text {singleturn }}$. This method indicates that the amount of emittance growth in the horizontal due to a $\beta$ mismatch consistent with these widths would be less than $10 \%$. Whereas, in the vertical, the emittance growth would be in the neighborhood of 30 to $40 \%$ (though including error bars could be as high as $185 \%$ ). So, using this method, the vertical emittance increase observed with the IPM on flattop is likely too much to be attributed solely to a $\beta$ mismatch (although this technique is limited as it only considers the special case where $\alpha=\alpha_{0}=0$ ). Yet, if the emittance growth seen between BtA and the flattop IPM is attributed to growth during the AGS cycle, than why is it greater at higher AGS intensities (i.e.-less scraping) than at lower intensities (see fig 4)?

The vertical IPM data from near injection, though somewhat suspect because of the uncertainty in the $\beta$ function value, is in relatively good agreement with the BtA values in figure 4 . It is also rather consistent with the estimate of $30-40 \%$ emittance growth due to a $\beta$ mismatch, especially in the cases where the beam is not heavily scraped. If this IPM data is valid though, then the vertical mismatch is not on the order indicated by the late IPM data in figure 4. Comparison of the BtA and IPM data in figure 4 suggest that there is an offset in the IPM data, that is worse at flattop than near injection. Although a model of how the IPM fits and acquires data agrees that there should be an offset that is smaller near injection than at flattop, the expected values of these offsets are about an order of magnitude smaller than what the actual IPM data suggests. $^{27}$

So, although the validity of the BtA emittance data is relatively well established, the AGS IPM flattop emittance data is hard to explain in physical terms as it doesn't seem to be solely a result of an injection mismatch, and is inversely proportional to AGS intensity not proportional to it. Therefore, although it seems pretty clear that the overall $\varepsilon_{R M S}$ coming out of the Booster was significantly smaller in FY'09 than in FY'06, it is hard to make a conclusive statement about the change in emittance late in the AGS between the two runs.

${ }^{27}$ L. Ahrens, "Measuring the Transverse Beam Size of the Beam with the AGS IPM: A Simple Exercise", 27 Aug 09 and figure 5. 